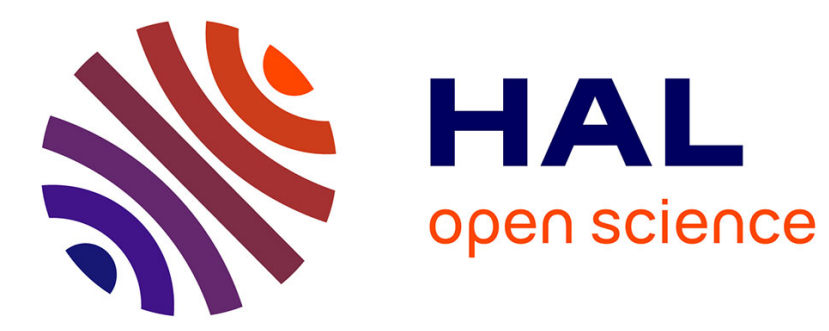

\title{
Couche limite dans un modèle de ferromagnétisme
}

Gilles Carbou, Pierre Fabrie, Olivier Guès

\section{To cite this version:}

Gilles Carbou, Pierre Fabrie, Olivier Guès. Couche limite dans un modèle de ferromagnétisme. Communications in Partial Differential Equations, 2002, 27 (7-8), pp.1467-1495. 10.1081/PDE-120005845 . hal-00295078

\section{HAL Id: hal-00295078 \\ https://hal.science/hal-00295078}

Submitted on 28 May 2019

HAL is a multi-disciplinary open access archive for the deposit and dissemination of scientific research documents, whether they are published or not. The documents may come from teaching and research institutions in France or abroad, or from public or private research centers.
L'archive ouverte pluridisciplinaire HAL, est destinée au dépôt et à la diffusion de documents scientifiques de niveau recherche, publiés ou non, émanant des établissements d'enseignement et de recherche français ou étrangers, des laboratoires publics ou privés. 


\title{
COUCHE LIMITE DANS UN MODÈLE DE FERROMAGNÉTISME
}

\author{
Gilles Carbou ${ }^{1}$, Pierre Fabrie $^{1}$ and Olivier Guès ${ }^{2}$ \\ 1 Mathématiques Appliquées de Bordeaux, UMR 5466 et Université Bordeaux 1, \\ 351 cours de la Libération, 33405 Talence cedex, France. \\ ${ }^{2}$ Laboratoire J.-A. Dieudonné, UMR CNRS 6621, Université de \\ Nice-Sophia-Antipolis, 06108 Nice, cedex 2, France.
}

\section{Résumé}

On étudie le comportement asymptotique des solutions des équations de LandauLifschitz lorsque le coefficient d'échange tend vers zéro. Une couche limite se forme alors au bord du matériau, et on décrit cette couche limite.

\begin{abstract}
In this paper we study the asymptotic limit of the solutions of Landau-Lifschitz equations as the exchange coefficient goes to zero. In order to perform this limit in regular spaces, we have to take into account the singularity induced by a boundary layer term.
\end{abstract}

\section{Introduction}

Le micromagnétisme est l'étude des phénomènes électromagnétiques dans les matériaux dits ferromagnétiques. Ces matériaux sont caractérisés par une magnétisation spontanée modélisée par le moment magnétique qui est un champ de vecteurs unitaires $u$ défini dans $\Omega$, le domaine où est confiné le matériau :

$$
u: \Omega \subset \mathbb{R}^{3} \longrightarrow \mathcal{S}^{2} \subset \mathbb{R}^{3} .
$$

Les variations de $u$ sont décrites par l'équation de Landau-Lifschitz : 


$$
\left\{\begin{array}{l}
\frac{\partial u}{\partial t}=u \wedge H_{e}-u \wedge\left(u \wedge H_{e}\right) \text { dans }[0, T] \times \Omega \\
\frac{\partial u}{\partial \mathbf{n}}=0 \text { sur }[0, T] \times \partial \Omega \\
u(0, x)=u_{0}(x) \text { sur } \Omega
\end{array}\right.
$$

où $\mathbf{n}$ est la normale au bord unitaire sortante, et où le champ efficace $H_{e}$ est donné par :

$$
H_{e}=\varepsilon^{2} \Delta u+\mathcal{H}(u) .
$$

On note $\mathcal{H}(u):=H_{\mid \Omega} \in L^{2}\left(\Omega ; \mathbb{R}^{3}\right)$ où le champ magnétique $H \in L^{2}\left(\mathbb{R}^{3} ; \mathbb{R}^{3}\right)$, appelé champ démagnétisant induit par u, est la solution du problème elliptique suivant,

$$
\left\{\begin{array}{l}
H \in L^{2}\left(\mathbb{R}^{3} ; \mathbb{R}^{3}\right), \\
\operatorname{rot} H=0 \text { dans } \mathbb{R}^{3}, \\
\operatorname{div}(H+\tilde{u})=0 \text { dans } \mathbb{R}^{3},
\end{array}\right.
$$

où $\tilde{u}$ désigne le prolongement de $u$ par zéro en dehors de $\Omega$. L'opérateur $\mathcal{H}($.$) , ainsi défini, opère de façon continue dans les espaces de Sobolev usuels$ $W^{m, p}(\Omega), 1<p<+\infty$.

Ce modèle appelé quasistationnaire, ainsi que d'autres modèles où (1.3) est remplacé par les équations de Maxwell dynamiques sont décrits en détail dans [4], [22] et [28].

Le coefficient $\varepsilon^{2}$ dans (1.2) est appelé coefficient d'échange. Etant très petit devant les autres grandeurs du problème, il est destiné à tendre vers zéro.

Lorsque le coefficient d'échange est fixé, Carbou et Fabrie démontrent dans [8] l'existence locale (et globale à donnée petite si $d=2$ ) d'une solution $u$ de $(1.1)$

$$
u \in \mathcal{C}\left([0, T] ; H^{2}(\Omega)\right) \cap L^{2}\left(0, T ; H^{3}(\Omega)\right) .
$$

Dans toute la suite, suivant la terminologie de [8], nous appellerons "solutions régulières" les solutions de (1.1) possèdant la régularité (1.4). Cependant, lorsque $\varepsilon$ tend vers zéro, les estimations obtenues dans [8] disparaissent, de sorte que l'intervalle de temps sur lequel l'existence de la solution régulière est garantie, tend vers 0 .

Le but de notre article est d'analyser plus précisemment le comportement de cette solution, lorsque $\varepsilon$ tend vers 0 . Pour des données initiales dans $H^{5}(\Omega)$, nous démontrons qu'en fait le temps d'existence de la solution régulière tend vers l'infini et que (en général) la norme $\|u(t, .)\|_{H^{2}(\Omega)}$ explose effectivement quand $\varepsilon \rightarrow 0$, pour tout $t>0$ fixé. Ceci est dù à la formation d'une couche 
limite au bord de l'ouvert $\Omega$, comme nous le verrons par la suite. En outre, nous montrons que la solution converge vers la solution du système hyperbolique obtenu formellement en prenant $\varepsilon=0$. Cela constitue une justification mathématique d'une approximation utilisée couramment dans les applications. Des phénomènes analogues apparaissent souvent dans des problèmes de perturbations visqueuses de systèmes hyperboliques (cf. [3], [23], [12], [13], [11], $[26])$.

\section{Enoncé des résultats}

On suppose que $\Omega$ est un ouvert borné régulier de $\mathbb{R}^{3}$. Pour toute donnée initiale $u_{0}$ satisfaisant $u_{0} \in H^{2}(\Omega),\left|u_{0}(x)\right|=1$ pour tout $x \in \Omega$, et $\partial u_{0} / \partial \mathbf{n}=0$ sur $\partial \Omega$, on sait d'après les résultats de l'article [8], que pour tout $\varepsilon>0$ fixé, il existe une unique solution régulière maximale de (1.1), notée $u^{\varepsilon}$ qui a pour temps maximal d'existence $T^{\varepsilon}$ et qui vérifie pour tout $T<T^{\varepsilon}$ :

$$
u^{\varepsilon} \in \mathcal{C}\left([0, T] ; H^{2}(\Omega)\right) \cap L^{2}\left(0, T ; H^{3}(\Omega)\right) .
$$

Le but de cet article est de préciser le comportement de la solution lorsque $\varepsilon \rightarrow 0$.

Lorsque l'on prend $\varepsilon=0$ dans l'équation (1.1), on obtient l'équation suivante

$$
\left\{\begin{array}{l}
\frac{\partial u^{0}}{\partial t}=u^{0} \wedge \mathcal{H}\left(u^{0}\right)-u^{0} \wedge\left(u^{0} \wedge \mathcal{H}\left(u^{0}\right)\right) \text { dans }[0,+\infty[\times \Omega \\
u^{0}(0, x)=u_{0}(x) \text { sur } \Omega
\end{array}\right.
$$

pour laquelle il est naturel de n'imposer aucune condition sur le bord $\mathbb{R} \times \partial \Omega$. Comme nous l'avons indiqué dans l'introduction, $\mathcal{H}$ opère sur les espaces de Sobolev (voir section 3):

Proposition 2.1 Pour tout entier $m \geq 0$, pour tout $p \in] 1,+\infty[, \mathcal{H}$ est linéaire continu de $W^{m, p}(\Omega)$ dans $W^{m, p}(\Omega)$.

Lorsque $m>3 / 2$, l'espace $W^{m, 2}(\Omega)=H^{m}(\Omega)$ est une algèbre et l'on peut donc interprèter (2.6) comme une équation différentielle ordinaire dans l'espace de Banach $H^{m}(\Omega)$. On déduit alors du théorème de Cauchy-Lipschitz l'existence locale d'une unique solution du problème 2.6. En fait, nous démontrons l'existence globale de ces solutions (section 4.2):

Théorème 2.1 Soit $m \geq 2$. Soit $u_{0} \in H^{m}(\Omega)$ satisfaisant $\left|u_{0}(x)\right|=1$ pour tout $x \in \Omega$. Il existe alors un unique $u^{0} \in C\left(\left[0,+\infty\left[; H^{m}(\Omega)\right)\right.\right.$ solution de $(2.6)$. En outre $\left|u^{0}(t, x)\right|=1$ pour tout $(t, x) \in\left[0,+\infty\left[\times \Omega\right.\right.$, et $u^{0} \in C^{\infty}\left(\left[0,+\infty\left[; H^{m}(\Omega)\right)\right.\right.$. 
Du point de vue des applications, lorsque l'on doit effectivement calculer $u^{\varepsilon}$, une approximation très courante en milieu industriel, consiste à remplacer le système (1.1) par le système (2.6), qui est plus "simple". A notre connaissance, il n'existait pas de résultat mathématique justifiant cette approximation, et l'une des motivations de ce travail est justement de préciser en quel sens $u^{0}$ est une bonne approximation de $u^{\varepsilon}$. Pour répondre à cette question, on doit tenir compte de la présence d'une couche limite qui se forme au voisinage du bord lorsque $\varepsilon \rightarrow 0$. C'est un résultat de ce type que nous allons maintenant décrire, obtenu sous la condition que la donnée initiale $u^{0}$ soit suffisamment régulière.

Fixons pour toute la suite de l'article une fonction $\varphi \in \mathcal{C}^{\infty}\left(\mathbb{R}^{d}, \mathbb{R}\right)$ telle que $\Omega=\{\varphi>0\}, \partial \Omega=\{\varphi=0\}$ et $|\nabla \varphi(x)|=1$ dans un voisinage $\mathcal{O}_{1}$ de $\partial \Omega^{1}$. Puisque $-\nabla \varphi(x)$ coïncide avec la normale unitaire sortante pour $x \in \partial \Omega$, nous étendons la notation et nous noterons dans toute la suite $\mathbf{n}(x):=-\nabla \varphi(x)$ $\left(\forall x \in \mathbb{R}^{3}\right)$. De façon analogue, nous noterons $\partial_{\mathbf{n}}$ le champ

$$
\partial_{\mathbf{n}}:=-\nabla_{x} \varphi \cdot \nabla_{x}=-\sum_{j=0}^{j=d} \partial_{x_{j}} \varphi(x) \partial_{x_{j}},
$$

à coefficients $\mathcal{C}^{\infty}$ sur $\mathbb{R}^{d}$, et qui coïncide sur $\partial \Omega$ avec la dérivée normale sortante.

Notre résultat principal est le suivant :

Théorème 2.2 Soit $u_{0} \in H^{5}(\Omega)$ vérifiant $\left|u_{0}\right|=1, \partial_{\mathbf{n}} u_{0 \mid \partial \Omega}=0$. Alors, si on note $T^{\varepsilon}$ le temps maximum d'existence de la solution régulière de l'équation (1.1) issue de la donnée initiale $u_{0}, \lim _{\varepsilon \rightarrow 0} T^{\varepsilon}=+\infty$. En outre, il existe une fonction $U \in L_{l o c}^{\infty}\left(\mathbb{R}_{t}^{+} ; H^{4}\left(\Omega_{x}\right) \otimes H^{4}\left(\mathbb{R}_{z}^{+}\right)\right)$telle que

$$
u^{\varepsilon}(t, x)=u^{0}(t, x)+\varepsilon U(t, x, \varphi(x) / \varepsilon)+\varepsilon v^{\varepsilon}(t, x)
$$

où pour tout $T<T^{\varepsilon}$, la fonction $v^{\varepsilon}$ est bornée dans $L^{\infty}\left(0, T ; H^{1}(\Omega)\right)$, et la fonction $\varepsilon v^{\varepsilon}$ est bornée dans $L^{\infty}\left(0, T ; H^{2}(\Omega)\right) \cap L^{2}\left(0, T ; H^{3}(\Omega)\right)$.

La fonction $U(t, x, z)$ introduite dans le théorème 2.2 est un profil de couche limite qui satisfait: $\lim _{z \rightarrow+\infty} U(t, x, z)=0$. Par un simple changement de variable, on en déduit que la solution maximale du problème (1.1) converge effectivement vers celle de (2.6) lorsque $\varepsilon \rightarrow 0$, au sens où d'une part, le temps d'existence de $u^{\varepsilon}$ tend vers l'infini et où, d'autre part, $u^{\varepsilon}$ converge vers $u^{0}$ dans $L^{2}\left([0, T] \times \mathbb{R}^{d}\right)$ pour tout $T>0$. De manière plus précise, on déduit du théorème 2.2 le corollaire suivant :

\footnotetext{
${ }^{1}$ On a donc pour $x \in \Omega \cap \mathcal{O}_{1}: \varphi(x)=\operatorname{dist}(x, \partial \Omega)$.
} 
Théorème 2.3 Supposons $u_{0} \in H^{5}(\Omega),\left|u_{0}\right|=1, \partial_{\mathbf{n}} u_{0 \mid \partial \Omega}=0$. Alors, pour tout $T>0$, il existe $\varepsilon_{T}>0$ tel que pour tout $\left.\left.\varepsilon \in\right] 0, \varepsilon_{T}\right]$, le problème (1.1) possède une unique solution $u^{\varepsilon}$ appartenant à $C\left([0, T] ; H^{2}(\Omega)\right)$ et celleci converge vers $u^{0}$ dans $C\left([0, T] ; H^{s}(\Omega)\right)$ pour $s<3 / 2$ lorsque $\varepsilon \rightarrow 0$, où $u^{0}$ est donné par le théorème 2.1. De plus, la solution $u^{\varepsilon}$ reste bornée dans $C\left([0, T] ; H^{3 / 2}(\Omega)\right)$ uniformément par rapport à $\varepsilon \rightarrow 0$.

Remarque 2.1 Il résulte aussi du théorème 2.2 que la quantité $\left\|u^{\varepsilon}(t, .)\right\|_{H^{2}(\Omega)}$ ne reste pas bornée (en général) quand $\varepsilon \rightarrow 0$, mais tend vers l'infini comme $\varepsilon^{-1}$. Cette remarque montre qu'il est inévitable que les majorations en norme $H^{2}(\Omega)$ de l'article $[8]$ explosent quand $\varepsilon \rightarrow 0$.

Comme on le fait remarquer dans [8], puisque $u$ est de norme 1, l'équation (1.1) est équivalente pour les solutions régulières à :

$$
\frac{\partial u}{\partial t}-\varepsilon^{2} \Delta u=\varepsilon^{2} u|\nabla u|^{2}+\varepsilon^{2} u \wedge \Delta u+u \wedge \mathcal{H}(u)-u \wedge(u \wedge \mathcal{H}(u)) .
$$

Cette équation a pour mérite d'isoler le terme dissipatif et c'est avec elle que nous allons travailler par la suite.

L'article est organisé de la manière suivante. La troisième partie est consacrée à la démonstration de quelques lemmes techniques donnant diverses formulations des inégalités de Sobolev et de Gagliardo-Nirenberg avec des normes adaptées. On montre aussi dans cette partie quelques propriétés de l'opérateur $\mathcal{H}$.

Dans la quatrième partie, on construit formellement le développement asymptotique de $u^{\varepsilon}$ et on montre que les termes qui le composent (à savoir $u^{0}$ et $U$ ) existent en tout temps et sont réguliers.

Le calcul des équations vérifiées par le reste $v^{\varepsilon}$ est fait dans la partie 5 .

Les estimations sur le reste font l'objet de la sixième partie.

Remarque 2.2 La théorie du ferromagnétisme a fait l'objet de nombreux travaux récents parmi lesquels on peut citer J. Joly, G. Metivier, J. Rauch [17], A. Visintin [27], G. Carbou [6], G. Carbou et P. Fabrie, [7], [8], D. Sanchez [25] pour l'étude qualitative de divers modèles et l'étude qualitative des solutions et S. Labbé [19], [20], P. Joly et O. Vacus [18], H. Haddar [14], H. Haddar et P. Joly [15] pour des travaux numériques.

\section{Quelques lemmes techniques}

\subsection{Normes équivalentes}

Les résultats contenus dans cette partie sont rappelés ou établis dans [8]. 
Lemme 3.1 Soit $\Omega$ un ouvert borné régulier de $\mathbb{R}^{3}$. Il existe une constante $C$ telle que pour tout $u \in H^{2}(\Omega)$ tel que $\partial_{\mathbf{n}} u=0$ sur $\partial \Omega$,

$$
\begin{gathered}
\|u\|_{H^{2}(\Omega)} \leq C\left(\|u\|_{L^{2}(\Omega)}^{2}+\|\Delta u\|_{L^{2}(\Omega)}^{2}\right)^{\frac{1}{2}}, \\
\|\nabla u\|_{H^{1}(\Omega)} \leq C\left(\|\nabla u\|_{L^{2}(\Omega)}^{2}+\|\Delta u\|_{L^{2}(\Omega)}^{2}\right)^{\frac{1}{2}},
\end{gathered}
$$

et pour $u \in H^{3}(\Omega)$ tel que $\partial_{\mathbf{n}} u=0$ sur $\partial \Omega$,

$$
\|\nabla u\|_{H^{2}(\Omega)} \leq C\left(\|\nabla u\|_{L^{2}(\Omega)}^{2}+\|\Delta u\|_{L^{2}(\Omega)}^{2}+\|\nabla \Delta u\|_{L^{2}(\Omega)}^{2}\right)^{\frac{1}{2}} .
$$

Remarque 3.1 On pourra consulter [1] et [2] pour l'inégalité (3.9), et [10] pour les inégalités (3.10) et (3.11).

Grâce au lemme précédent et grâce aux inégalités classiques d'interpolation, on peut re-écrire les inégalité de Sobolev et de Gagliardo-Nirenberg sous la forme :

Lemme 3.2 Soit $\Omega$ un ouvert borné régulier de $\mathbb{R}^{3}$. Il existe une constante $C$ telle que pour tout $u \in H^{2}(\Omega)$ tel que $\partial_{\mathbf{n}} u=0$ sur $\partial \Omega$,

$$
\begin{gathered}
\|u\|_{L^{\infty}(\Omega)} \leq C\left(\|u\|_{L^{2}}^{2}+\|\Delta u\|_{L^{2}}^{2}\right)^{\frac{1}{2}} \\
\|\nabla u\|_{L^{6}(\Omega)} \leq C\left(\|u\|_{L^{2}}^{2}+\|\Delta u\|_{L^{2}}^{2}\right)^{\frac{1}{2}}, \\
\|\nabla u\|_{L^{4}(\Omega)}^{2} \leq C\|u\|_{L^{\infty}(\Omega)}\left(\|u\|_{L^{2}}^{2}+\|\Delta u\|_{L^{2}(\Omega)}^{2}\right)^{\frac{1}{2}},
\end{gathered}
$$

et pour tout $u \in H^{3}(\Omega)$ tel que $\partial_{\mathbf{n}} u=0$ sur $\partial \Omega$,

$$
\left\|D^{2} u\right\|_{L^{3}(\Omega)} \leq C\left(\left(\|u\|_{L^{2}}^{2}+\|\Delta u\|_{L^{2}}^{2}\right)^{\frac{1}{2}}+\left(\|u\|_{L^{2}}^{2}+\|\Delta u\|_{L^{2}}^{2}\right)^{\frac{1}{4}}\|\nabla \Delta u\|_{L^{2}}^{\frac{1}{2}}\right) .
$$

\section{$3.2 \quad$ Etude de l'opérateur $\mathcal{H}$}

On note $\mathbf{H}$ l'opérateur qui à $u \in L^{2}\left(\Omega ; \mathbb{R}^{3}\right)$ associe $\mathbf{H}(u) \in L^{2}\left(\mathbb{R}^{3}, \mathbb{R}^{3}\right)$, unique solution dans $L^{2}\left(\mathbb{R}^{3}, \mathbb{R}^{3}\right)$ du problème elliptique suivant

$$
\operatorname{rot} \mathbf{H}(u)=0 \quad, \quad \operatorname{div}(\mathbf{H}(u)+\tilde{u})=0
$$

où $\tilde{u}$ prolonge $u$ par zéro en dehors de $\Omega$, de sorte que suivant les notations de l'introduction $\mathcal{H}(u)=\mathbf{H}(u)_{\mid \Omega}$. 
Proposition 3.1 Pour tout $p \in] 1,+\infty[$ et tout $k \geq 1$, il existe une constante $C_{p, k}$ telle que

$$
\forall u \in W^{k, p}(\Omega), \quad\left\|\left.\mathbf{H}(u)\right|_{\Omega}\right\|_{W^{k, p}(\Omega)} \leq C_{p, k}\|u\|_{W^{k, p}(\Omega)} .
$$

En particulier, pour tout $k \geq 1$, il existe une constante $C_{k}$ telle que

$$
\forall u \in H^{k}(\Omega),\left\|\left.\mathbf{H}(u)\right|_{\Omega}\right\|_{H^{k}(\Omega)} \leq C_{k}\|u\|_{H^{k}(\Omega)} .
$$

Preuve. Dans [8], on montre que l'opérateur $\mathcal{H}$ est borné dans tous les $W^{1, p}(\Omega)$, pour $1<p<+\infty$. La démonstration est exactement la même ici :

1. On écrit que $\mathbf{H}(u)=-\nabla \psi$, où $\psi$ vérifie :

$$
\begin{cases}-\Delta \psi=-\operatorname{div} u & \operatorname{dans} \Omega, \\ -\Delta \psi=0 & \operatorname{dans} \Omega^{\prime}, \\ {[\psi]_{\mid \partial \Omega}=0, \quad\left[\partial_{\mathbf{n}} \psi\right]_{\left.\right|_{\partial \Omega}}=u \cdot \mathbf{n},} & \end{cases}
$$

où $\Omega^{\prime}={ }^{C} \bar{\Omega}$ et $\left.[\psi]\right|_{\partial \Omega}$ est le saut de $\psi$ à travers $\partial \Omega$.

2. On considère un ouvert borné régulier $\Omega_{2}$ contenant $\bar{\Omega}$ et on relève le saut de $\psi$ en considérant $\psi_{1} \in W^{k+1, p}\left(\Omega_{2} \backslash \bar{\Omega}\right)$ vérifiant :

$$
\left.\psi_{1}\right|_{\partial \Omega}=0,\left.\psi_{1}\right|_{\partial \Omega_{2}^{\prime}}=0,\left.\partial_{\mathbf{n}} \psi_{1}\right|_{\partial \Omega_{2}^{\prime}}=0,\left.\partial_{\mathbf{n}} \psi_{1}\right|_{\partial \Omega}=u \cdot \mathbf{n},
$$

où $\Omega_{2}^{\prime}$ est le complémentaire de $\Omega_{2}$. Par surjectivité de la trace, l'existence de $\psi_{1}$ est évidente.

On est donc ramené à trouver $\varphi$ solution de

$$
\begin{cases}-\Delta \varphi=-\operatorname{div} u & \text { dans } \Omega, \\ -\Delta\left(\varphi+\psi_{1}\right)=0 & \operatorname{dans} \Omega_{2} \backslash \bar{\Omega} \\ -\Delta \varphi=0 & \operatorname{dans} \Omega_{2}^{\prime} \\ {\left.[\varphi]\right|_{\partial \Omega_{2}}=\left.0 \quad\left[\partial_{\mathbf{n}} \varphi\right]\right|_{\partial \Omega_{2}}=0,} & \end{cases}
$$

On note alors

$$
\begin{aligned}
& f_{1}=-\operatorname{div} u \mathbf{1}_{\Omega}, \\
& f_{2}=\Delta \psi_{1} \mathbf{1}_{\Omega_{2} \backslash \bar{\Omega}}, \\
& f=f_{1}+f_{2},
\end{aligned}
$$


Avec ces notations, $\varphi$ est solution de

$$
-\Delta \varphi=f \quad \text { dans } \mathbb{R}^{d}
$$

3. On applique alors les résultats classiques de régularité des solutions de problèmes elliptiques (cf. [21] page 196) et on obtient le résultat désiré.

En introduisant la transformation de Fourier notée $\mathcal{F}(\cdot)$ définie par

$$
(\mathcal{F} u)(\xi)=\int_{\mathbb{R}^{d}} e^{-i x \cdot \xi} u(x) d x
$$

on obtient pour $\mathbf{H}(u)$ l'expression suivante

$$
\mathbf{H}(u)(x)=-(2 \pi)^{-d} \int_{\mathbb{R}^{d}} e^{i x \cdot \xi} \frac{(\xi \cdot(\mathcal{F} \tilde{u})(\xi))}{|\xi|^{2}} \xi d \xi
$$

Introduisons une fonction de troncature $\chi \in \mathcal{C}_{0}^{\infty}\left(\mathbb{R}^{d}, \mathbb{R}\right), \operatorname{supp}(\chi) \subset\{|\xi|<1\}$, $\chi(\xi) \equiv 1$ sur un voisinage de $0 \in \mathbb{R}^{d}$. On écrit

$$
\mathbf{H}(u)=\mathbf{p}(D)(\tilde{u})+R(\tilde{u})
$$

où $\mathbf{p}(D)$ est un cas particulier ${ }^{2}$ d'opérateur pseudo-différentiel matriciel de symbole classique matriciel $\mathbf{p} \in S^{0}\left(\mathbb{R}_{x}^{d} \times \mathbb{R}_{\xi}^{d} ; \mathcal{M}_{3}(\mathbb{R})\right)$, défini par $\mathbf{p}(\xi) \cdot v=$ $-(1-\chi(\xi))(\xi \cdot v) \xi|\xi|^{-2}$, qui agit sur une fonction $v \in\left(L^{2}\left(\mathbb{R}^{d}\right)\right)^{3}$ suivant la formule

$$
\begin{aligned}
\mathbf{p}(D) v(x) & :=-(2 \pi)^{-d} \int_{\mathbb{R}^{d}} e^{i x \cdot \xi}(1-\chi(\xi)) \frac{(\xi \cdot(\mathcal{F} v)(\xi))}{|\xi|^{2}} \xi d \xi \\
& =(2 \pi)^{-d} \int_{\mathbb{R}^{d}} e^{i x \cdot \xi} \mathbf{p}(\xi)(\mathcal{F} v)(\xi) d \xi,
\end{aligned}
$$

et où $R(\tilde{u})=-(2 \pi)^{-d} \int_{\mathbb{R}^{d}} e^{i x \cdot \xi}(\xi \cdot(\mathcal{F} \tilde{u})(\xi)) \xi|\xi|^{-2} \chi(\xi) d x$, est infiniment régularisant (continu de $L^{2}\left(\mathbb{R}^{d}\right)$ dans $H^{s}\left(\mathbb{R}^{d}\right)$ pour tout $s \in \mathbb{R}$ ) (voir [9], [16]). La proposition 3.1 entraine que $\mathbf{p}(D)$ satisfait la propriété de transmission ([9], [16], [5]) pour l'ouvert $\Omega$. Le résultat général suivant, qui s'applique au cas de $\mathbf{p}(D)$, est dû à Guy Métivier [24]

Théorème 3.1 Soit $P(x, D)$ un opérateur pseudo-différentiel classique de degré 0 sur $\mathbb{R}^{N}$ possédant la propriété de transmission pour $\Omega$, ouvert borné régulier de $\mathbb{R}^{N}$. Pour tout $s>N / 2$ il existe $c_{s}>0$ telle que pour tout $u \in H^{s}(\Omega)$ :

$$
\left\|(P(x, D)(\tilde{u}))_{\mid \Omega}\right\|_{L^{\infty}(\Omega)} \leq c_{s}\|u\|_{L^{\infty}(\Omega)} \ln \left(1+\frac{\|u\|_{H^{s}(\Omega)}}{\|u\|_{L^{\infty}(\Omega)}}\right) .
$$

\footnotetext{
${ }^{2}$ C'est un simple multiplicateur de Fourier, son symbole $p(\xi)$ ne dépendant pas de $x$.
} 
En appliquant ce résultat à $\mathbf{P}(D)$ on en déduit que pour tout $s>3 / 2$, il existe une constante $c_{s}>0$ telle que pour tout $u \in H^{s}(\Omega)$,

$$
\|\mathcal{H}(u)\|_{L^{\infty}(\Omega)} \leq c_{s}\|u\|_{L^{\infty}(\Omega)} \ln \left(1+\frac{\|u\|_{H^{s}(\Omega)}}{\|u\|_{L^{\infty}(\Omega)}}\right)
$$

estimation qui permet d'établir l'existence globale des solutions du problème limite $(\varepsilon=0)$.

\section{Construction du développement asympto- tique de $u^{\varepsilon}$}

\subsection{Ansatz}

On cherche un développement asymptotique de $u^{\varepsilon}$ et de $H^{\varepsilon}=\mathcal{H}\left(u^{\varepsilon}\right)$ sous la forme :

$$
\begin{aligned}
& u^{\varepsilon}(t, x)=U^{0}\left(t, x, \frac{\varphi(x)}{\varepsilon}\right)+\varepsilon U^{1}\left(t, x, \frac{\varphi(x)}{\varepsilon}\right)+\ldots \\
& H^{\varepsilon}(t, x)=H^{0}\left(t, x, \frac{\varphi(x)}{\varepsilon}\right)+\varepsilon H^{1}\left(t, x, \frac{\varphi(x)}{\varepsilon}\right)+\ldots
\end{aligned}
$$

où les fonctions $U^{i}$ et $H^{i}$ se décomposent sous la forme :

$$
V(t, x, z)=\bar{V}(t, x)+\widetilde{V}(t, x, z),
$$

avec $\bar{V}(t, x)=\lim _{z \underset{\widetilde{V}}{\longrightarrow}+\infty} V(t, x, z)$.

On suppose que $\widetilde{V}$, ainsi que toutes ses dérivées partielles aussi bien en $x$ qu'en $z$ tendent vers zéro lorsque $z$ tend vers $+\infty$. En fait, le terme $\overline{U^{i}}$ est un terme intérieur d'ordre $\varepsilon^{i}$ tandis que le terme $\widetilde{U^{i}}$ est le terme de couche limite d'ordre $\varepsilon^{i}$.

On remplace alors $u^{\varepsilon}$ et $H^{\varepsilon}$ dans les équation (2.8) et (1.3) par leurs développements asymptotiques et on identifie formellement les coefficients des différentes puissances de $\varepsilon$.

Ordre -1. La condition au bord dans (1.1) donne $\partial_{\mathbf{n}} \varphi U_{z}^{0}=0$ pour $x \in \partial \Omega$ et $z=0$

L'équation (1.3) donne :

$$
\nabla \varphi \wedge H_{z}^{0}=0 \text { et } \nabla \varphi \cdot\left(H_{z}^{0}+U_{z}^{0}\right)=0 .
$$

On obtient donc que $H_{z}^{0}=-\left(U_{z}^{0} \cdot \mathbf{n}\right) \mathbf{n}$, et comme $\overline{H^{0}}$ et $\overline{U^{0}}$ ne dépendent pas de $z$, ceci peut s'écrire :

$$
\widetilde{H^{0}}=-\left(\widetilde{U^{0}} \cdot \mathbf{n}\right) \mathbf{n}+K(t, x)
$$


où $K$ est une constante d'intégration. Maintenant, puisque $\widetilde{H^{0}}$ et $\widetilde{U^{0}}$ doivent tendre vers zéro lorsque $z$ tend vers $+\infty$, on obtient que la constante d'intégration $K$ est nulle.

Ainsi,

$$
\left.\widetilde{H^{0}}=-\widetilde{\left(U^{0}\right.} \cdot \mathbf{n}\right) \mathbf{n}
$$

Ordre 0. De l'équation (2.8), il vient :

$\frac{\partial U^{0}}{\partial t}-|\nabla \varphi|^{2} U_{z z}^{0}=|\nabla \varphi|^{2}\left|U_{z}^{0}\right|^{2} U^{0}+|\nabla \varphi|^{2} U^{0} \wedge U_{z z}^{0}+U^{0} \wedge H^{0}-U^{0} \wedge\left(U^{0} \wedge H^{0}\right)$.

On fait tendre $z$ vers $+\infty$ dans cette équation et on obtient :

$$
\frac{\partial \overline{U^{0}}}{\partial t}=\overline{U^{0}} \wedge \overline{H^{0}}-\overline{U^{0}} \wedge\left(\overline{U^{0}} \wedge \overline{H^{0}}\right)
$$

D'autre part, de (1.3), il vient :

$$
\begin{aligned}
& \operatorname{rot} H^{0}+\nabla \varphi \wedge H_{z}^{1}=0 \\
& \operatorname{div}\left(H^{0}+U^{0}\right)+\nabla \varphi \cdot\left(U_{z}^{1}+H_{z}^{1}\right)=0 .
\end{aligned}
$$

En faisant tendre $z$ vers $+\infty$, on obtient $\operatorname{rot} \overline{H^{0}}=0$ et $\operatorname{div}\left(\overline{H^{0}}+\overline{U^{0}}\right)=0$, ce qui se traduit par :

$$
\overline{H^{0}}=\mathcal{H}\left(\overline{U^{0}}\right) .
$$

En soustrayant de l'équation sur $U^{0}$ l'équation obtenue pour $\overline{U^{0}}$, on obtient l'équation suivante pour $\widetilde{U^{0}}$ :

$$
\begin{aligned}
\frac{\partial \widetilde{U^{0}}}{\partial t}-|\nabla \varphi|^{2} \widetilde{U_{z z}^{0}}= & |\nabla \varphi|^{2}\left|\widetilde{U_{z}^{0}}\right|^{2}\left(\widetilde{U^{0}}+\overline{U^{0}}\right)+|\nabla \varphi|^{2}\left(\widetilde{U^{0}}+\overline{U^{0}}\right) \wedge \widetilde{U_{z z}^{0}} \\
& +\widetilde{U^{0}} \wedge \overline{H^{0}}+\overline{U^{0}} \wedge \widetilde{H^{0}}+\widetilde{U^{0}} \wedge \widetilde{H^{0}} \\
& -\widetilde{U^{0}} \wedge\left(U^{0} \wedge H^{0}\right)-\overline{U^{0}} \wedge\left(\widetilde{U^{0}} \wedge H^{0}\right)-\overline{U^{0}} \wedge\left(\overline{U^{0}} \wedge \widetilde{H^{0}}\right) .
\end{aligned}
$$

De la condition aux limites à l'ordre -1 , on a que $\widetilde{U_{z}^{0}}=0$ pour $z=0$. On prend donc

$$
\widetilde{U^{0}} \equiv 0
$$

qui est solution de l'équation obtenue.

On écrit maintenant la condition de Neumann à l'ordre 0, et on obtient :

$$
\partial_{\mathbf{n}} U^{0}+\partial_{\mathbf{n}} \varphi U_{z}^{1}=0
$$


pour $z=0$ et $x \in \partial \Omega$. Puisque $\partial_{\mathbf{n}} \varphi=-1$ sur $\partial \Omega$, et comme $\widetilde{U^{0}}$ est nul, on a :

$$
U_{z}^{1}=\partial_{\mathbf{n}} \overline{U^{0}} \text { en } z=0 .
$$

Cette égalité n'est a priori vraie que pour $x \in \partial \Omega$, mais on l'étend pour $x$ dans le voisinage $\mathcal{O}_{1} \mathrm{du}$ bord.

Ordre 1. On écrit l'ordre 1 pour l'équation (2.8), en remarquant que les termes en $U_{z}^{0}$ sont nuls puisque $\widetilde{U^{0}} \equiv 0$.

$\frac{\partial U^{1}}{\partial t}-|\nabla \varphi|^{2} U_{z z}^{1}=|\nabla \varphi|^{2} U^{0} \wedge U_{z z}^{1}+U^{0} \wedge H^{1}+U^{1} \wedge H^{0}+$ termes trilinéaires.

En faisant tendre $z$ vers $+\infty$, on obtient alors :

$\frac{\partial \overline{U^{1}}}{\partial t}=\overline{U^{0}} \wedge \overline{H^{1}}+\overline{U^{1}} \wedge \overline{H^{0}}-\overline{U^{1}} \wedge\left(\overline{U^{0}} \wedge \overline{H^{0}}\right)-\overline{U^{0}} \wedge\left(\overline{U^{1}} \wedge \overline{H^{0}}\right)-\overline{U^{0}} \wedge\left(\overline{U^{0}} \wedge \overline{H^{1}}\right)$

Si on regarde l'ordre 1 dans (1.3), on obtient :

$$
\begin{aligned}
& \operatorname{div}\left(H^{1}+U^{1}\right)+\nabla \varphi \cdot\left(U_{z}^{2}+H_{z}^{2}\right)=0, \\
& \operatorname{rot} H^{1}+\nabla \varphi \wedge H_{z}^{2}=0,
\end{aligned}
$$

et en faisant tendre $z$ vers $+\infty$, les termes en $U_{z}^{2}$ tendent vers zéro, et on obtient que

$$
\overline{H^{1}}=\mathcal{H}\left(\overline{U^{1}}\right) .
$$

Ainsi, l'équation définissant $\overline{U^{1}}$ est linéaire, et si la donnée initiale est nulle, la solution est nulle. On peut donc prendre :

$$
\overline{U^{1}} \equiv 0 \text { et } \overline{H^{1}} \equiv 0 .
$$

L'équation en $\widetilde{U^{1}}$ devient donc :

$\frac{\partial \widetilde{U^{1}}}{\partial t}-|\nabla \varphi|^{2} \widetilde{U_{z z}^{1}}=|\nabla \varphi|^{2} \overline{U^{0}} \wedge \widetilde{U_{z z}^{1}}+\overline{U^{0}} \wedge \widetilde{H^{1}}+\widetilde{U^{1}} \wedge \overline{H^{0}}-$ termes trilinéaires.

D'autre part, en ne gardant que ce qui concerne la partie en tilde dans (4.21), on a :

$$
\nabla \varphi \cdot\left(\widetilde{U_{z}^{1}}+\widetilde{H_{z}^{1}}\right)=0 \text { et } \nabla \varphi \wedge \widetilde{H_{z}^{1}}=0 .
$$

En intégrant en $z$ cette équation et en annulant la constante d'intégration comme on l'a déjà fait, on obtient que :

$$
\left.\widetilde{H^{1}}=-\widetilde{\left(U^{1}\right.} \cdot \mathbf{n}\right) \mathbf{n} .
$$


Joint aux conditions initiales en $z$ que l'on a vu plus haut, ceci définit complètement tous les termes du développement asymptotique, comme nous allons le voir maintenant. On remarque qu'à l'ordre 1 , l'opérateur $\mathcal{H}$ se localise ce qui est un phénomène analogue à ce qui se passe pour une couche mince (voir [6], [14] et $[15])$.

\subsection{Etude des profils}

\subsubsection{Existence de $\overline{U^{0}}$}

On définit $\overline{U^{0}}$ comme étant la solution de l'équation différentielle ordinaire en temps suivante :

$$
\left\{\begin{array}{l}
\frac{\partial \overline{U^{0}}}{\partial t}=\overline{U^{0}} \wedge \overline{H^{0}}-\overline{U^{0}} \wedge\left(\overline{U^{0}} \wedge \overline{H^{0}}\right) \text { sur }[0, T[\times \Omega \\
\overline{U^{0}}(t=0, x)=u_{0}(x) \operatorname{sur} \Omega \\
\overline{H^{0}}=\mathcal{H}\left(\overline{U^{0}}\right)
\end{array}\right.
$$

On rappelle que $u_{0} \in H^{5}(\Omega)$ et que $\left|u_{0}(x)\right|=1$ pour tout $x \in \Omega$.

On a alors la proposition suivante :

Proposition 4.1 L'équation (4.27) admet une et une seule solution qui est dans $\mathcal{C}^{\infty}\left(\mathbb{R}^{+} ; H^{5}(\Omega)\right)$.

\section{Preuve :}

Premier pas : l'opérateur $\mathcal{H}$ est un opérateur linéaire continu de $H^{5}(\Omega)$ dans $H^{5}(\Omega)$. De plus, cet espace est une algèbre. On peut donc appliquer le théorème de Cauchy-Lipschitz à l'équation (4.27), ce qui assure l'existence et l'unicité de la solution maximale sur un intervalle de temps de la forme $\left[0, T_{e}[\right.$.

En multipliant ponctuellement l'équation par $\overline{U^{0}}$, on s'aperçoit que $\frac{\partial\left|\overline{U^{0}}\right|^{2}}{\partial t}=0$, ce qui montre que $\overline{U^{0}}$ reste de norme constante égale à 1 , puisque $u_{0}$ vérifie cette propriété.

Deuxième pas : majoration en norme $H^{2}$.

Montrons maintenant que la solution maximale est bornée dans $L^{\infty}\left(0, T ; H^{2}(\Omega)\right)$. En dérivant deux fois l'équation dans les variables d'espace, on a :

$$
\begin{gathered}
\frac{\partial}{\partial t}\left(\frac{\partial^{2} \overline{U^{0}}}{\partial x_{i} \partial x_{j}}\right)=\frac{\partial^{2} \overline{U^{0}}}{\partial x_{i} \partial x_{j}} \wedge \mathcal{H}\left(\overline{U^{0}}\right)+\frac{\partial \overline{U^{0}}}{\partial x_{i}} \wedge \frac{\partial \mathcal{H}\left(\overline{U^{0}}\right)}{\partial x_{j}}+\frac{\partial \overline{U^{0}}}{\partial x_{j}} \wedge \frac{\partial \mathcal{H}\left(\overline{U^{0}}\right)}{\partial x_{i}}+\overline{U^{0}} \wedge \frac{\partial^{2} \mathcal{H}\left(\overline{U^{0}}\right)}{\partial x_{i} \partial x_{j}} \\
+ \text { termes trilinéaires. }
\end{gathered}
$$


On intègre sur $[0, t]$ cette équation et on majore en norme. On obtient alors sachant que $\left|U^{0}\right|=1$ :

$$
\begin{aligned}
\left\|\overline{U^{0}}(t)\right\|_{H^{2}} \leq & \left\|u_{0}\right\|_{H^{2}}+\int_{0}^{t}\left\|\overline{U^{0}}(s)\right\|_{H^{2}}\left\|\mathcal{H}\left(\overline{U^{0}}(s)\right)\right\|_{L^{\infty}} d s \\
& +\int_{0}^{t}\left(\left\|\mathcal{H}\left(\overline{U^{0}}(s)\right)\right\|_{H^{2}}+\left\|\overline{U^{0}}(s)\right\|_{H^{1}}\left\|\mathcal{H}\left(\overline{U^{0}}(s)\right)\right\|_{H^{1}}\right) d s \\
& +C \int_{0}^{t}\left(\left\|\overline{U^{0}}(s)\right\|_{H^{2}}\left\|\mathcal{H}\left(\overline{U^{0}}(s)\right)\right\|_{L^{\infty}}+\left\|\overline{U^{0}}(s)\right\|_{H^{1}}^{2}\left\|\mathcal{H}\left(\overline{U^{0}}(s)\right)\right\|_{L^{\infty}}\right) d s \\
& +C \int_{0}^{t}\left(\left\|\overline{U^{0}}(s)\right\|_{H^{1}}\left\|\mathcal{H}\left(\overline{U^{0}}(s)\right)\right\|_{H^{1}}+\left\|\mathcal{H}\left(\overline{U^{0}}(s)\right)\right\|_{H^{2}}\right) d s .
\end{aligned}
$$

Or, comme $\left\|\overline{U^{0}}\right\|_{L^{\infty}}=1$, on sait que

$$
\begin{aligned}
& \left\|\mathcal{H}\left(\overline{U^{0}}\right)\right\|_{L^{\infty}} \leq C \ln \left(\left\|\overline{U^{0}}\right\|_{H^{2}}+1\right), \\
& \left\|\overline{U^{0}}\right\|_{H^{1}}^{2} \leq C\left\|\overline{U^{0}}\right\|_{H^{2}} .
\end{aligned}
$$

Par ailleurs, puisque

$$
\left\|\mathcal{H}\left(\overline{U^{0}}\right)\right\|_{H^{1}(\Omega)} \leq C\left\|\overline{U^{0}}\right\|_{H^{1}(\Omega)}
$$

on a :

$$
\left\|\overline{U^{0}}(t)\right\|_{H^{2}} \leq\left\|u_{0}\right\|_{H^{2}}+C \int_{0}^{t}\left[1+\ln \left(\left\|\overline{U^{0}}(s)\right\|_{H^{2}}+1\right)\right]\left\|\overline{U^{0}}(s)\right\|_{H^{2}} d s,
$$

ce qui montre par théorème classique de comparaison que $\overline{U^{0}}(t)$ reste borné en norme $H^{2}(\Omega)$ sur tout intervalle de temps borné.

\section{Troisième pas : majoration dans $H^{3}$.}

En dérivant trois fois en espace l'équation vérifiée par $\overline{U^{0}}$, en intégrant entre 0 et $T$, en majorant en norme et en remarquant que $\left|\overline{U^{0}}(t, x)\right|=1$ presque partout, on obtient que :

$$
\begin{aligned}
\left\|\overline{U^{0}}(t)\right\|_{H^{3}} \leq & \left\|u_{0}\right\|_{H^{3}}+C \int_{0}^{t}\left\|\overline{U^{0}}(s)\right\|_{H^{3}}\left\|\mathcal{H}\left(\overline{U^{0}}\right)(s)\right\|_{L^{\infty}} d s \\
& +\int_{0}^{t}\left\|\overline{U^{0}}(s)\right\|_{H^{2}}\left\|\mathcal{H}\left(\overline{U^{0}}\right)(s)\right\|_{H^{1}}+C \int_{0}^{t}\left\|\overline{U^{0}}(s)\right\|_{H^{1}}\left\|\mathcal{H}\left(\overline{U^{0}}\right)(s)\right\|_{H^{2}} d s \\
& +C \int_{0}^{t}\left\|\mathcal{H}\left(\overline{U^{0}}\right)(s)\right\|_{H^{3}} d s+\text { termes trilinéaires. }
\end{aligned}
$$


Or, on a montré dans le pas précédent que $\overline{U^{0}} \in L^{\infty}\left(0, T ; H^{2}(\Omega)\right)$ pour tout $T \leq T_{\varepsilon}$. De plus, $\mathcal{H}$ est un opérateur borné de $H^{s}$ dans $H^{s}$. En particulier, $\mathcal{H}\left(\overline{U^{0}}\right)$ est borné dans $L^{\infty}\left(0, T ; H^{2}(\Omega)\right)$, donc $\mathcal{H}\left(\overline{U^{0}}\right)$ est borné dans $L^{\infty}(] 0, T[\times \Omega)$. On obtient ainsi que

$$
\left\|\overline{U^{0}}\right\|_{H^{3}}(t) \leq\left\|u_{0}\right\|_{H^{3}}+K t+K \int_{0}^{t}\left\|\overline{U^{0}}(s)\right\|_{H^{3}} d s,
$$

ce qui montre grâce au lemme de Gronwall que $\overline{U^{0}}(t)$ reste borné en norme $H^{3}(\Omega)$ sur tout intervalle de temps borné.

\section{Quatrième pas : conclusion.}

De la même manière que dans le troisième pas, on montre successivement que $\overline{U^{0}}(t)$ reste borné en norme $H^{4}(\Omega)$ puis $H^{5}(\Omega)$ sur tout intervalle de temps borné. Par théorème classique, puisque ce que l'on vient de prouver empêche toute explosion en temps fini, on obtient que $\overline{U^{0}}$ est définie sur tout $\mathbb{R}^{+}$à valeurs dans $H^{5}(\Omega)$.

\subsection{Existence de $\widetilde{U^{1}}$}

Pour $x \in \mathcal{O}_{1}$, on cherche $\widetilde{U^{1}}(t, x, z)$ solution de l'équation linéaire suivante, où $\widetilde{H^{1}}=-\left(\widetilde{U^{1}} \cdot \mathbf{n}\right) \mathbf{n}$.

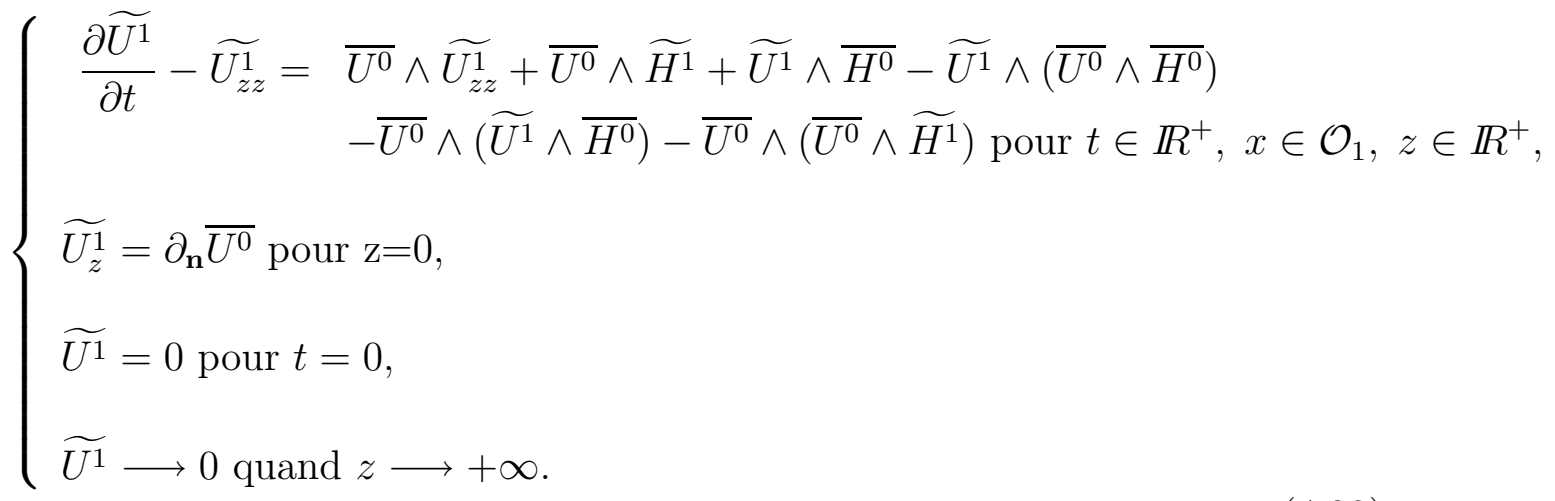

Proposition 4.2 Il existe une et une seule solution de (4.28) vérifiant

$$
\widetilde{U^{1}} \in \mathcal{C}\left(\mathbb{R}_{t}^{+} ; H^{4}\left(\mathcal{O}_{1}\right) \otimes H^{4}\left(\mathbb{R}^{+}\right)\right) \cap L^{2}\left(\mathbb{R}_{t}^{+} ; H^{4}\left(\mathcal{O}_{1}\right) \otimes H^{5}\left(\mathbb{R}^{+}\right)\right) .
$$

Preuve : après avoir relevé la condition au bord pour se ramener à un problème homogène, on va résoudre le problème pour $z \in[0, L]$ avec condition de Neuman en $z=L$, via une approximation de Galerkin. On obtiendra alors des estimations sur $w$ et sur $\frac{\partial w}{\partial t}$ en norme $L^{\infty}\left(0, T ; H^{2}(0, L)\right) \cap L^{2}\left(0, T ; H^{3}(0, L)\right)$. 
Les estimations obtenues seront indépendantes de $L$ ce qui nous permettra de passer à la limite lorsque $L$ tend vers $+\infty$, pour construire alors une solution pour $z \in \mathbb{R}^{+}$. Le gain de régularité sera obtenu en inversant l'équation et en écrivant $w_{z z}$ en fonction de $\frac{\partial w}{\partial t}$.

Premier pas : on se ramène à un problème homogène en relevant la condition aux limites en $z=0$. Pour cela on introduit une fonction $\zeta$ de la seule variable $z$, telle que $\zeta^{\prime}(0)=1$, telle que le support de $\zeta$ est contenu dans $[0,1]$, et on pose $\theta(t, x, z)=\zeta(z) \partial_{\mathbf{n}} \overline{U^{0}}$. On cherche alors $\widetilde{U^{1}}$ sous la forme $\widetilde{U^{1}}=w+\theta$ où $w$ vérifie des conditions aux limites homogènes. L'équation aux dérivées partielles en $(t, z)$, paramétrée par $x$, vérifiée par $w$ s'écrit :

$w_{t}(t, x, z)-w_{z z}(t, x, z)=\overline{U^{0}}(t, x) \wedge w(t, x, z)+G(t, x)(w(t, x, z))+F(t, x, z)$,

où $G \in \mathcal{M}_{3}\left(\mathcal{C}^{\infty}\left(\mathbb{R}_{t}^{+}\right) \otimes H^{4}(\Omega)\right)$ et où la fonction $F$ est $\mathcal{C}^{\infty}$ dans la variable $z$.

\section{Deuxième pas :}

On remarque d'abord que ce système étant linéaire, il y a bien évidement unicité dans la classe considérée. Pour établir l'existence on résout le probleme précédent sur $] 0, L[$ avec les conditions aux limites suivantes :

$$
\begin{aligned}
& U_{z}^{1}=\partial_{\mathbf{n}} \overline{U^{0}} \text { pour } \mathrm{z}=0 \\
& U_{z}^{1}=0 \text { pour } \mathrm{z}=\mathrm{L}
\end{aligned}
$$

on obtient des estimations indépendantes de $L$, puis on fait tendre $L$ vers l'infini.

On prend donc une approximation de Galerkin en la variable $z$ sur $[0, L]$ de l'équation (4.29) avec les conditions aux limites (4.30) et sur cette approximation, on fait les estimations a priori suivantes :

Estimation $H^{1}$.

En multipliant l'équation (4.29) par $-w_{z z}$ et en intégrant par parties dans la variable $z$ on obtient

$$
\frac{d}{d t}\left\|w_{z}\right\|_{L^{2}}^{2}+\left\|w_{z z}\right\|_{L^{2}}^{2} \leq\|G\|_{L^{\infty}}\left\|w_{z}\right\|_{L^{2}}^{2}+\left\|F_{z}\right\|_{L^{2}}\left\|w_{z}\right\|_{L^{2}}
$$

soit, par le lemme de Gronwall,

$$
\left\|w_{z}(t)\right\|_{L^{2}}^{2}+\int_{0}^{t}\left\|w_{z z}(\tau)\right\|_{L^{2}}^{2} d \tau \leq C(t) .
$$

Estimation $H^{2}$.

Multipliant l'équation (4.29) par $w_{z z z z}$, en remarquant que $\overline{U^{0}}$ ne dépend pas de $z$, il vient 


$$
\frac{d}{d t}\left\|w_{z z}\right\|_{L^{2}}^{2}+\left\|w_{z z z}\right\|_{L^{2}}^{2} \leq\|G\|_{L^{\infty}}\left\|w_{z}\right\|_{L^{2}}\left\|w_{z z z}\right\|_{L^{2}}+\left\|F_{z}\right\|_{L^{2}}\left\|w_{z z z}\right\|_{L^{2}},
$$

soit, par le lemme de Gronwall, en utilisant les estimations précédentes

$$
\left\|w_{z z}(t)\right\|_{L^{2}}^{2}+\int_{0}^{t}\left\|w_{z z z}(\tau)\right\|_{L^{2}}^{2} d \tau \leq C(t) .
$$

En prenant le supremum en $x$ dans les inégalités précédentes on obtient des estimations dans $L^{\infty}\left(\mathcal{O}_{1}\right) \otimes H^{2}$. Pour obtenir des estimation $L^{\infty}$ en $x$ sur les dérivées, il suffit des raisonner comme précédemment sur l'équation dérivée.

Equation sur $\frac{\partial w}{\partial t}$.

On dérive en temps l'approximation de Galerkin de (4.29), et en notant $W=$ $\frac{\partial w}{\partial t}$, on obtient que $W$ vérifie :

$$
\begin{cases}W_{t}(t, x, z)-W_{z z}(t, x, z)= & \overline{U^{0}}(t, x) \wedge W_{z z}(t, x, z)+G(t, x)(W(t, x, z)) \\ & +\frac{\partial F}{\partial t}(t, x, z)+\frac{\partial \overline{U^{0}}}{\partial t}(t, x) \wedge w_{z z}(t, x, z)+\frac{\partial G}{\partial t}(t, x)(w(t, x, z)), \\ W_{z}(0)=W_{z}(L)=0 . & \end{cases}
$$

Estimation $H^{1}$ sur $\frac{\partial w}{\partial t}$.

On multiplie l'équation (4.31) par $W_{z z}$ et on intègre en $z$ sur $[0, L]$. On obtient alors :

$$
\begin{aligned}
\frac{1}{2} \frac{d}{d t}\left\|W_{z}\right\|_{L^{2}}^{2}+ & \left\|W_{z z}\right\|_{L^{2}}^{2} \leq\|G\|_{L^{\infty}}\left\|W_{z}\right\|_{L^{2}}^{2}+ \\
& \left\|\frac{\partial F}{\partial t}(t, x, z)+\frac{\partial \overline{U^{0}}}{\partial t}(t, x) \wedge w_{z z}(t, x, z)+\frac{\partial G}{\partial t}(t, x)(w(t, x, z))\right\|_{L^{2}}\left\|W_{z z}\right\|_{L^{2}},
\end{aligned}
$$

et en absorbant le terme en $\left\|W_{z z}\right\|_{L^{2}}$ du membre de droite, on obtient que

$$
\begin{aligned}
\frac{d}{d t}\left\|W_{z}\right\|_{L^{2}}^{2}+\left\|W_{z z}\right\|_{L^{2}}^{2} \leq & \|G\|_{L^{\infty}}\left\|W_{z}\right\|_{L^{2}}^{2} \\
& +\left(\left\|\frac{\partial F}{\partial t}\right\|_{L^{2}}+\left\|\frac{\partial \overline{U^{0}}}{\partial t}\right\|_{L^{\infty}}\left\|w_{z z}\right\|_{L^{2}}+\left\|\frac{\partial G}{\partial t}\right\|_{L^{\infty}}\|w\|_{L^{2}}\right)^{2} .
\end{aligned}
$$


Estimation $H^{2}$ sur $\frac{\partial w}{\partial t}$.

On multiplie l'approximation de Galerkin l'équation (4.31) par $W_{z z z z}$ et on intègre en $z$ sur $[0, L]$. On obtient alors :

$$
\begin{aligned}
\frac{d}{d t}\left\|W_{z z}\right\|_{L^{2}}^{2}+\left\|W_{z z z}\right\|_{L^{2}}^{2} \leq & \|G\|_{L^{\infty}}\left\|W_{z z}\right\|_{L^{2}}^{2} \\
& +\left(\left\|\frac{\partial^{2} F}{\partial t \partial z}\right\|_{L^{2}}+\left\|\frac{\partial \overline{U^{0}}}{\partial t}\right\|_{L^{\infty}}\left\|w_{z z z}\right\|_{L^{2}}+\left\|\frac{\partial G}{\partial t}\right\|_{L^{\infty}}\left\|w_{z}\right\|_{L^{2}}\right)\left\|W_{z z z}\right\|_{L^{2}},
\end{aligned}
$$

ce qui donne après absorbtion du terme en $\left\|W_{z z z}\right\|_{L^{2}}$ qu'il existe une fonction $M(t)$ qui est $L^{1}$ en temps telle que

$$
\frac{d}{d t}\left\|W_{z z}\right\|_{L^{2}}^{2}+\left\|W_{z z z}\right\|_{L^{2}}^{2} \leq\|G\|_{L^{\infty}}\left\|W_{z z}\right\|_{L^{2}}^{2}+M(t)
$$

où $M(t)=\left(\left\|\frac{\partial^{2} F}{\partial t \partial z}\right\|_{L^{2}}+\left\|\frac{\partial \overline{U^{0}}}{\partial t}\right\|_{L^{\infty}}\left\|w_{z z z}\right\|_{L^{2}}+\left\|\frac{\partial G}{\partial t}\right\|_{L^{\infty}}\left\|w_{z}\right\|_{L^{2}}\right)^{2}$.

Troisième pas. On passe à la limite dans l'approximation de Galerkin. Les majorations obtenus ne dépendent pas de $L$ (notamment car $\zeta$ est à support compact fixé). On pousse donc $L$ vers $+\infty$ et on obtient donc l'existence d'une fonction $w$ qui satisfait (4.29) et qui vérifie en outre que pout tout $T$,

$$
\left\{\begin{array}{l}
w \in L^{\infty}\left(\mathcal{O}_{1} ; L^{\infty}\left([0, T] ; H^{2}\left(\mathbb{R}^{+}\right)\right) \cap L^{2}\left([0, T] ; H^{3}\left(\mathbb{R}^{+}\right)\right)\right) \\
\frac{\partial w}{\partial t} \in L^{\infty}\left(\mathcal{O}_{1} ; L^{\infty}\left(0, T ; H^{2}\left(\mathbb{R}^{+}\right)\right) \cap L^{2}\left(0, T ; H^{3}\left(\mathbb{R}^{+}\right)\right)\right)
\end{array}\right.
$$

On inverse alors l'endomorphisme de $\mathbb{R}^{3}$ qui à $\xi$ associe $\xi+\overline{U^{0}}(t, x) \wedge \xi$. On peut ainsi exprimer $w_{z z}$ en fonction de $\frac{\partial w}{\partial t}$, ce qui montre que $w_{z z}$ a la même régularité que $\frac{\partial w}{\partial t}$. Ainsi, on obtient que :

$$
w \in L^{\infty}\left(\mathcal{O}_{1} ; L^{\infty}\left([0, T] ; H^{4}\left(\mathbb{R}^{+}\right)\right) \cap L^{2}\left([0, T] ; H^{5}\left(\mathbb{R}^{+}\right)\right)\right) .
$$

Conclusion. Pour obtenir la régularité en $x$ de $w$, on dérive l'équation (4.29) par rapport à $x_{i}$ et on procède de la même manière que précédemment pour obtenir des majorations sur les dérivées en espace de $w$, ce qui permet de conclure la preuve de la proposition. 


\section{Equation vérifiée par le reste}

Soit $\psi \in \mathcal{C}_{0}^{\infty}\left(\mathbb{R}^{3}, \mathbb{R}\right)$, telle que $\operatorname{supp}(\psi) \subset \mathcal{O}_{1}$ et $\psi \equiv 1$ sur un voisinage de $\partial \Omega$.

Soit $\Theta \in L^{\infty}\left(\mathbb{R}^{+} ; H^{4}(\Omega)\right)$ tel que $\partial_{\mathbf{n}} \Theta(t, x)=-\partial_{\mathbf{n}} \widetilde{U^{1}}(t, x, 0)$ sur $\mathbb{R}^{+} \times \partial \Omega$.

On pose

$$
u^{\varepsilon}(t, x)=\overline{U^{0}}(t, x)+\varepsilon \psi(x) \widetilde{U^{1}}\left(t, x, \frac{\varphi(x)}{\varepsilon}\right)+\varepsilon \Theta(t, x)+\varepsilon v^{\varepsilon}(t, x),
$$

et l'on écrit $H^{\varepsilon}:=\mathcal{H}\left(u^{\varepsilon}\right)$ sous la forme

$H^{\varepsilon}(t, x)=\overline{H^{0}}(t, x)+\varepsilon \psi(x) \widetilde{H^{1}}\left(t, x, \frac{\varphi(x)}{\varepsilon}\right)+\varepsilon R^{\varepsilon}(t, x)+\varepsilon \mathcal{H}(\Theta)(t, x)+\varepsilon \mathcal{H}\left(v^{\varepsilon}\right)(t, x)$,

où $R^{\varepsilon}(t, x)=\left(\mathcal{H}\left(\psi \widetilde{U^{1}}\right)-\psi(x) \widetilde{H^{1}}\right)(t, x)$.

On note :

$$
\begin{aligned}
& a^{\varepsilon}(t, x)=\overline{U^{0}}(t, x)+\varepsilon \psi(x) \widetilde{U^{1}}\left(t, x, \frac{\varphi(x)}{\varepsilon}\right)+\varepsilon \Theta(t, x), \\
& K^{\varepsilon}:=\mathcal{H}\left(a^{\varepsilon}\right)=\overline{H^{0}}(t, x)+\varepsilon \psi(x) \widetilde{H^{1}}\left(t, x, \frac{\varphi(x)}{\varepsilon}\right)+\varepsilon R^{\varepsilon}+\varepsilon \mathcal{H}(\Theta)(t, x) .
\end{aligned}
$$

On rappelle que

$$
\overline{H^{0}}=\mathcal{H}\left(\overline{U^{0}}\right) \text { et } \widetilde{H^{1}}=-\left(\widetilde{U^{1}} \cdot \mathbf{n}\right) \mathbf{n} .
$$

Un calcul algébrique donne que $v^{\varepsilon}$ vérifie l'équation suivante :

$$
\left\{\begin{array}{l}
\partial_{\mathbf{n}} v^{\varepsilon}=0 \text { sur } \partial \Omega \\
\frac{\partial v^{\varepsilon}}{\partial t}-\varepsilon^{2} \Delta v^{\varepsilon}=T_{1}+\ldots+T_{8}+F^{\varepsilon} \text { sur }[0, T] \times \Omega \\
v^{\varepsilon}(0, x)=0 \text { dans } \Omega
\end{array}\right.
$$


où

$$
\begin{aligned}
& T_{1}=\varepsilon^{4} v^{\varepsilon}\left|\nabla v^{\varepsilon}\right|^{2}, \\
& T_{2}=\varepsilon^{3}\left(a^{\varepsilon}\left|\nabla v^{\varepsilon}\right|^{2}+2 v^{\varepsilon}\left(\nabla a^{\varepsilon}, \nabla v^{\varepsilon}\right)\right), \\
& T_{3}=\varepsilon^{2}\left(v^{\varepsilon}\left|\nabla a^{\varepsilon}\right|^{2}+2 a^{\varepsilon}\left(\nabla v^{\varepsilon}, \nabla a^{\varepsilon}\right)\right), \\
& T_{4}=+\varepsilon^{2} v^{\varepsilon} \wedge \Delta a^{\varepsilon}+\varepsilon^{2} a^{\varepsilon} \wedge \Delta v^{\varepsilon}+\varepsilon^{3} v^{\varepsilon} \wedge \Delta v^{\varepsilon}, \\
& T_{5}=v^{\varepsilon} \wedge K^{\varepsilon}+a^{\varepsilon} \wedge \mathcal{H}\left(v^{\varepsilon}\right)+\varepsilon v^{\varepsilon} \wedge \mathcal{H}\left(v^{\varepsilon}\right), \\
& T_{6}=-\left[a^{\varepsilon} \wedge\left(a^{\varepsilon} \wedge \mathcal{H}\left(v^{\varepsilon}\right)\right)+a^{\varepsilon} \wedge\left(v^{\varepsilon} \wedge K^{\varepsilon}\right)+v^{\varepsilon} \wedge\left(a^{\varepsilon} \wedge K^{\varepsilon}\right)\right], \\
& T_{7}=-\varepsilon\left[v^{\varepsilon} \wedge\left(v^{\varepsilon} \wedge K^{\varepsilon}\right)+v^{\varepsilon} \wedge\left(a^{\varepsilon} \wedge \mathcal{H}\left(v^{\varepsilon}\right)\right)+a^{\varepsilon} \wedge\left(v^{\varepsilon} \wedge \mathcal{H}\left(v^{\varepsilon}\right)\right)\right], \\
& T_{8}=-\varepsilon^{2} v^{\varepsilon} \wedge\left(v^{\varepsilon} \wedge \mathcal{H}\left(v^{\varepsilon}\right)\right) .
\end{aligned}
$$

On a noté

$F^{\varepsilon}:=-\varepsilon^{-1}\left(\partial_{t} a^{\varepsilon}-\varepsilon^{2} \Delta a^{\varepsilon}-\varepsilon^{2} a^{\varepsilon} \wedge \Delta a^{\varepsilon}-\left|\nabla a^{\varepsilon}\right|^{2} \cdot a^{\varepsilon}-a^{\varepsilon} \wedge K^{\varepsilon}+a^{\varepsilon} \wedge\left(a^{\varepsilon} \wedge K^{\varepsilon}\right)\right)$.

ce qui s'écrit encore

$$
\begin{aligned}
F^{\varepsilon}= & -\frac{\partial \Theta}{\partial t}+\varepsilon A_{1}+\varepsilon a^{\varepsilon}\left|\nabla a^{\varepsilon}\right|^{2}+\varepsilon a^{\varepsilon} \wedge A_{1}+\varepsilon^{2} \Psi \widetilde{U^{1}} \wedge \Delta a^{\varepsilon} \\
& +a^{\varepsilon} \wedge R^{\varepsilon}+\varepsilon \widetilde{U^{1}} \wedge \widetilde{H^{1}}+\varepsilon \Theta \wedge \widetilde{H^{1}}-a^{\varepsilon} \wedge\left(a^{\varepsilon} \wedge R^{\varepsilon}\right) \\
& -\varepsilon \overline{U^{0}} \wedge\left(\left(\widetilde{U^{1}}+\Theta\right) \wedge \widetilde{H^{1}}\right)-\varepsilon\left(\widetilde{U^{1}}+\Theta\right) \wedge\left(\overline{U^{0}} \wedge \widetilde{H^{1}}\right) \\
& -\varepsilon\left(\widetilde{U^{1}}+\Theta\right) \wedge\left(\left(\widetilde{U^{1}}+\Theta\right) \wedge \overline{H^{0}}\right)-\varepsilon^{2}\left(\widetilde{U^{1}}+\Theta\right) \wedge\left(\left(\widetilde{U^{1}}+\Theta\right) \wedge \widetilde{H^{1}}\right),
\end{aligned}
$$

avec

$$
\begin{aligned}
A_{1}= & \Delta a^{\varepsilon}-\frac{1}{\varepsilon} \Psi \widetilde{U_{z z}^{1}} \\
= & \Delta \overline{U^{0}}+\psi \Delta \varphi \widetilde{U_{z}^{1}}+2(\nabla \psi, \nabla \varphi) \widetilde{U_{z}^{1}}+2 \psi\left(\nabla \varphi, \nabla \widetilde{U_{z}^{1}}\right) \\
& +\varepsilon \Delta \psi \widetilde{U^{1}}+\varepsilon \psi \Delta \widetilde{U^{1}}+2 \varepsilon \nabla \psi \nabla \widetilde{U^{1}}+\varepsilon \Delta \Theta .
\end{aligned}
$$

D'après les résultats de régularités obtenus sur les profils on obtient la proposition ci-dessous : 
Proposition 5.1 Pour tout $p, 1<p \leq+\infty$, et tout $T>0$, il existe des constantes $C_{p}$ indépendantes de $\varepsilon$ telle que pour tout $\varepsilon>0$ et tout $\left.\left.t \in\right] 0, T\right]$,

$$
\begin{aligned}
& \left\|a^{\varepsilon}(t, .)\right\|_{W^{1, p}} \leq C_{p}, \\
& \varepsilon\left\|D^{2} a^{\varepsilon}(t, .)\right\|_{L^{p}} \leq C_{p} .
\end{aligned}
$$

Pour tout $p, 1<p<+\infty$, et tout $T>0$, il existe des constantes $C_{p}$ indépendantes de $\varepsilon$ telle que pour tout $\varepsilon>0$ et tout $t \in] 0, T]$,

$$
\varepsilon^{2}\left\|\nabla \Delta a^{\varepsilon}(t, .)\right\|_{L^{p}} \leq C_{p} .
$$

Pour tout $p, 1<p<+\infty$, et tout $T>0$, il existe des constantes $C_{p}$ indépendantes de $\varepsilon$ telle que pour tout $\varepsilon>0$ et tout $t \in] 0, T]$,

$$
\begin{gathered}
\left\|K^{\varepsilon}(t, .)\right\|_{W^{1, p}} \leq C_{p}, \\
\|F(t, .)\|_{W^{1, p}} \leq C_{p} .
\end{gathered}
$$

Preuve : D'après l'expression de $a^{\varepsilon}$ il suffit de regarder, compte tenu de la régularité de $\overline{U^{0}}$, la régularité de $\widetilde{U^{1}}$. La fonction $(t, x, z) \mapsto \widetilde{U^{1}}(t, x, z)$ est continue en temps à valeurs dans $H^{4}\left(\mathcal{O}_{1}\right) \times H^{4}(\mathbb{R})$. Elle est donc continue en temps à valeurs dans $W^{3, p}\left(\mathcal{O}_{1} \times \mathbb{R}\right)$ pour tout $p$ fini et également continue en temps à valeurs dans $\mathcal{C}_{b}^{2}\left(\mathcal{O}_{1} \times \mathbb{R}\right)$. Ceci donne les estimations sur $a^{\varepsilon}$. L'estimation sur $K^{\varepsilon}=\mathcal{H}\left(a^{\varepsilon}\right)$ résulte de celle concernant $a^{\varepsilon}$ et de la continuité de l'opérateur $\mathcal{H}$ sur $W^{1, p}$ pour $p$ fini. En particulier, si $p>3$, on obtient que $K^{\varepsilon}$ est borné dans $L^{\infty}$. L'estimation sur $F^{\varepsilon}$ s'obtient par un raisonnement analogue.

\section{Estimations sur le reste}

Nous allons ici donner une estimation sur le reste $v^{\varepsilon}$. En fait, on établit par là même un résultat d'existence, ce qui donne une autre démonstration que celle proposée dans [8]. Il faut noter que cette nouvelle preuve d'existence est obtenue pour des données initiales $H^{5}(\Omega)$ et non des données $H^{3}(\Omega)$. Cette exigence de régularité supplémentaire est classique dans ce type de méthodes utilisant un développement asymptotique. Ces estimations sur $v^{\varepsilon}$ sont faites sur une approximation de Galerkin, ce qui permet de justifier toutes les intégrations par parties effectuées. L'espace d'approximation est construit sur la base des fonctions propres du laplacien de domaine $\{u \in$ $\left.H^{2}(\Omega), \nabla u \cdot \mathbf{n}_{\mid \partial \Omega}=0\right\}$ pour l'équation de Landau Lifschitz. Pour l'équation sur le champ magnétique, on ne fait pas d'approximation particulière. On introduit simplement $H_{n}=\mathcal{H}\left(v_{n}^{\varepsilon}\right)$ partout où cela est nécessaire. Afin de rendre 
les calculs plus lisibles, on ne détaille pas cette étape. Dans toute la suite, on note $\mathbf{Q}(t)$ (ou simplement $\mathbf{Q}$ ) la quantité

$$
\mathbf{Q}(t):=\left(\left\|v_{n}^{\varepsilon}(t, .)\right\|_{L^{2}}^{2}+\left\|\nabla v_{n}^{\varepsilon}(t, .)\right\|_{L^{2}}^{2}+\left\|\varepsilon \Delta v_{n}^{\varepsilon}(t, .)\right\|_{L^{2}}^{2}\right) .
$$

Cette quantité existe sur un intervalle maximal $\left[0, T_{n, \varepsilon}^{*}\left[\right.\right.$, où $0<T_{n, \varepsilon}^{*} \leq \infty$. Le but de ce paragraphe est de démontrer qu'il existe $\varepsilon_{T}>0$ suffisamment petit, tel que $\mathbf{Q}$ existe sur $[0, T]$ si $\varepsilon \leq \varepsilon_{T}$, indépendamment de $n$. On démontre également les estimations annoncées dans le résultat principal. La méthode employée consiste à fixer un réel $T>0$ arbitraire et à montrer grâce à des estimations d'énergie (établies par intégration par parties) que $\mathbf{Q}(t)$ satisfait, sur $[0, T] \cap\left[0, T_{n, \varepsilon}^{*}[\right.$, une inéquation différentielle de la forme

$$
\mathbf{Q}^{\prime}(t) \leq C_{T}(1+\mathbf{Q}(t))+\varepsilon^{1 / 2} P_{T}(\mathbf{Q}(t)) \quad, \quad t \in[0, T] \cap\left[0, T_{n, \varepsilon}^{*}[,\right.
$$

où $C_{T}$ est une constante et $P_{T}$ un polynôme, tous deux étant indépendants de $n$ et $\varepsilon$ (mais dépendants de $T$ ). Cette inégalité entraine alors que $T_{n, \varepsilon}^{*} \geq T$ dès que $\left.\varepsilon \in] 0, \varepsilon_{T}\right]$ où $\varepsilon_{T}>0$ est une constante suffisamment petite. Il en résulte que $\mathbf{Q}(t)$ satisfait (6.36) sur tout l'intervalle $[0, T]$, et le théorème principal s'en déduit de manière classique.

Dans les estimations qui suivent, nous utiliserons souvent les inégaliés suivantes que nous rappelons pour le confort du lecteur:

$$
\|u\|_{L 6(\Omega)} \leq C\|u\|_{H^{1}(\Omega)},\|u\|_{L^{4}(\Omega)} \leq C\|u\|_{L^{2}(\Omega)}^{1 / 4}\|u\|_{H^{1}(\Omega)}^{3 / 4},\|u\|_{L 3(\Omega)} \leq C\|u\|_{L^{2}(\Omega)}^{1 / 2}\|u\|_{H^{1}(\Omega)}^{1 / 2} .
$$

\section{Estimation $L^{2}$.}

On multiplie (5.32) par $v^{\varepsilon}$ et on intègre sur $\Omega$. Les intégrations par parties sont licites sur les approximants de Galerkin. On obtient :

$$
\frac{1}{2} \frac{d}{d t}\left(\left\|v^{\varepsilon}\right\|_{L^{2}}^{2}\right)+\varepsilon^{2}\left\|\nabla v^{\varepsilon}\right\|_{L^{2}}^{2} \leq \int_{\Omega}\left(T_{1}+\ldots+T_{8}+F^{\varepsilon}\right) v^{\varepsilon} .
$$

On majore alors chaque $\int_{\Omega} T_{i} v^{\varepsilon}$. Dans chaque estimation, on majore d'abord chaque intégrale par inégalités de Hölder, puis, dans un deuxième temps, en utilisant les injections de Sobolev :

$$
\begin{aligned}
\left|\int_{\Omega} T_{1} v^{\varepsilon}\right| & \leq \varepsilon^{4}\left\|v^{\varepsilon}\right\|_{L^{\infty}}^{2}\left\|\nabla v^{\varepsilon}\right\|_{L^{2}}^{2} \\
& \leq C \varepsilon^{2}\left\|v^{\varepsilon}\right\|_{H^{1}}^{2}\left\|\varepsilon v^{\varepsilon}\right\|_{H^{2}}^{2} \leq C \varepsilon^{2} \mathbf{Q}^{2}
\end{aligned}
$$




$$
\begin{aligned}
& \left|\int_{\Omega} T_{2} v^{\varepsilon}\right| \leq \varepsilon^{3}\left\|a^{\varepsilon}\right\|_{L^{\infty}}\left\|v^{\varepsilon}\right\|_{L^{3}}\left\|\nabla v^{\varepsilon}\right\|_{L^{3}}^{2}+2 \varepsilon^{3}\left\|v^{\varepsilon}\right\|_{L^{3}}^{2}\left\|\nabla a^{\varepsilon}\right\|_{L^{\infty}}\left\|\nabla v^{\varepsilon}\right\|_{L^{3}} \\
& \leq C \varepsilon^{2}\left\|v^{\varepsilon}\right\|_{L^{2}}^{1 / 2}\left\|v^{\varepsilon}\right\|_{H^{1}}^{1 / 2}\left\|v^{\varepsilon}\right\|_{H^{1}}\left\|\varepsilon v^{\varepsilon}\right\|_{H^{2}} \\
& +C \varepsilon^{5 / 2}\left\|v^{\varepsilon}\right\|_{L^{2}}\left\|v^{\varepsilon}\right\|_{H^{1}}\left\|v^{\varepsilon}\right\|_{H^{1}}^{1 / 2}\left\|\varepsilon v^{\varepsilon}\right\|_{H^{2}}^{1 / 2} \leq C \varepsilon^{2} \mathbf{Q}^{3 / 2} \\
& \left|\int_{\Omega} T_{3} v^{\varepsilon}\right| \leq \varepsilon^{2}\left\|v^{\varepsilon}\right\|_{L^{2}}^{2}\left\|\nabla a^{\varepsilon}\right\|_{L^{\infty}}^{2}+2 \varepsilon^{2}\left\|a^{\varepsilon}\right\|_{L^{\infty}}\left\|v^{\varepsilon}\right\|_{L^{2}}\left\|\nabla v^{\varepsilon}\right\|_{L^{2}}\left\|\nabla a^{\varepsilon}\right\|_{L^{\infty}} \\
& \leq C \varepsilon^{2} \mathbf{Q} \\
& \left|\int_{\Omega} T_{4} v^{\varepsilon}\right| \leq \varepsilon^{2}\left\|v^{\varepsilon}\right\|_{L^{2}}\left\|\nabla a^{\varepsilon}\right\|_{L^{\infty}}\left\|\nabla v^{\varepsilon}\right\|_{L^{2}} \\
& \leq C \varepsilon^{2}\left\|v^{\varepsilon}\right\|_{L^{2}}\left\|\nabla v^{\varepsilon}\right\|_{L^{2}} \leq C \varepsilon^{2} \mathbf{Q} \\
& \left|\int_{\Omega} T_{5} v^{\varepsilon}\right| \leq\left\|a^{\varepsilon}\right\|_{L^{\infty}}\left\|v^{\varepsilon}\right\|_{L^{2}}\left\|\mathcal{H}\left(v^{\varepsilon}\right)\right\|_{L^{2}} \\
& \leq C\left\|v^{\varepsilon}\right\|_{L^{2}}^{2} \\
& \left|\int_{\Omega} T_{6} v^{\varepsilon}\right| \leq\left\|a^{\varepsilon}\right\|_{L^{\infty}}^{2}\left\|v^{\varepsilon}\right\|_{L^{2}}\left\|\mathcal{H}\left(v^{\varepsilon}\right)\right\|_{L^{2}}+\left\|a^{\varepsilon}\right\|_{L^{\infty}}\left\|K^{\varepsilon}\right\|_{L^{\infty}}\left\|v^{\varepsilon}\right\|_{L^{2}}^{2} \\
& \leq C\left\|v^{\varepsilon}\right\|_{L^{2}}\left\|\mathcal{H}\left(v^{\varepsilon}\right)\right\|_{L^{2}}+C\left\|v^{\varepsilon}\right\|_{L^{2}}^{2} \leq C\left\|v^{\varepsilon}\right\|_{L^{2}}^{2} \\
& \left|\int_{\Omega} T_{7} v^{\varepsilon}\right| \leq \varepsilon\left\|v^{\varepsilon}\right\|_{L^{4}}^{2}\left\|\mathcal{H}\left(v^{\varepsilon}\right)\right\|_{L^{2}} \\
& \leq C \varepsilon\left\|v^{\varepsilon}\right\|_{L^{2}}^{3 / 2}\|v\|_{H^{1}}^{3 / 2} \leq C \varepsilon \mathbf{Q}^{3 / 2} \\
& \left|\int_{\Omega} T_{8} v^{\varepsilon}\right|=0 \\
& \left|\int_{\Omega} F^{\varepsilon} v^{\varepsilon}\right| \leq\left\|F^{\varepsilon}\right\|_{L^{2}}\left\|v^{\varepsilon}\right\|_{L^{2}} \\
& \leq C\left(1+\left\|v^{\varepsilon}\right\|_{L^{2}}^{2}\right)
\end{aligned}
$$

En utilisant les majorations (5.33), (5.35), on obtient qu'il existe une constante $C$ telle que

$$
\frac{d}{d t}\left(\left\|v^{\varepsilon}\right\|_{L^{2}}^{2}\right) \leq C\left(1+\left\|v^{\varepsilon}\right\|_{L^{2}}^{2}\right)+\varepsilon P(\mathbf{Q})
$$

où $P$ est une fonction à croissance polynômiale.

\section{Estimation $H^{1}$.}

En multipliant une approximation de Galerkin de l'équation (5.32) par $\Delta v^{\varepsilon}$ et en intégrant par parties, on obtient que :

$$
\frac{1}{2} \frac{d}{d t}\left(\left\|\nabla v^{\varepsilon}\right\|_{L^{2}}^{2}\right)+\varepsilon^{2}\left\|\Delta v^{\varepsilon}\right\|_{L^{2}}^{2} \leq \int_{\Omega}\left(T_{1}+\ldots+T_{8}+F^{\varepsilon}\right) \Delta v^{\varepsilon}
$$


et on majore chaque terme de droite de la manière suivante :

$$
\begin{aligned}
\left|\int_{\Omega} T_{1} \Delta v^{\varepsilon}\right| \leq & \varepsilon^{4}\left\|v^{\varepsilon}\right\|_{L^{6}}\left\|\Delta v^{\varepsilon}\right\|_{L^{2}}\left\|\nabla v^{\varepsilon}\right\|_{L^{6}}^{2} \\
\leq & C \varepsilon\left\|v^{\varepsilon}\right\|_{H^{1}}\left\|\varepsilon v^{\varepsilon}\right\|_{H^{2}}^{3} \\
\leq & C \varepsilon \mathbf{Q}^{2} \\
\left|\int_{\Omega} T_{2} \Delta v^{\varepsilon}\right| \leq & \varepsilon^{3}\left\|a^{\varepsilon}\right\|_{L^{\infty}}\left\|\nabla v^{\varepsilon}\right\|_{L^{4}}^{2}\left\|\Delta v^{\varepsilon}\right\|_{L^{2}} \\
& +2 \varepsilon^{3}\left\|v^{\varepsilon}\right\|_{L^{6}}\left\|\nabla v^{\varepsilon}\right\|_{L^{3}}\left\|\nabla a^{\varepsilon}\right\|_{L^{\infty}}\left\|\Delta v^{\varepsilon}\right\|_{L^{2}} \\
\leq & C \varepsilon^{\frac{1}{2}}\left\|v^{\varepsilon}\right\|_{H^{1}}^{\frac{1}{2}}\left\|\varepsilon v^{\varepsilon}\right\|_{H^{2}}^{\frac{5}{2}}+C \varepsilon^{\frac{3}{2}}\left\|v^{\varepsilon}\right\|_{H^{1}}^{\frac{3}{2}}\left\|\varepsilon v^{\varepsilon}\right\|_{H^{2}}^{\frac{3}{2}} \\
\leq & C \varepsilon^{\frac{1}{2}} \mathrm{Q}^{\frac{3}{2}}
\end{aligned}
$$




$$
\begin{aligned}
& \left|\int_{\Omega} T_{3} \Delta v^{\varepsilon}\right| \leq \varepsilon\left\|v^{\varepsilon}\right\|_{L^{2}}\left\|\nabla a^{\varepsilon}\right\|_{L^{\infty}}^{2}\left\|\varepsilon \Delta v^{\varepsilon}\right\|_{L^{2}} \\
& +2 \varepsilon\left\|a^{\varepsilon}\right\|_{L^{\infty}}\left\|\nabla v^{\varepsilon}\right\|_{L^{2}}\left\|\nabla a^{\varepsilon}\right\|_{L^{\infty}}\left\|\varepsilon \Delta v^{\varepsilon}\right\|_{L^{2}} \\
& \leq C \varepsilon \mathbf{Q} \\
& \left|\int_{\Omega} T_{4} \Delta v^{\varepsilon}\right| \leq \varepsilon^{2}\left\|v^{\varepsilon}\right\|_{L^{6}}\left\|\nabla \Delta a^{\varepsilon}\right\|_{L^{3}}\left\|\nabla v^{\varepsilon}\right\|_{L^{2}} \\
& \leq C\left\|v^{e}\right\|_{H^{1}}^{2} \\
& \leq C \mathrm{Q} \\
& \left|\int_{\Omega} T_{5} \Delta v^{\varepsilon}\right| \leq\left\|v^{\varepsilon}\right\|_{L^{6}}\left\|\nabla K^{\varepsilon}\right\|_{L^{3}}\left\|\nabla v^{\varepsilon}\right\|_{L^{2}}+\left\|a^{\varepsilon}\right\|_{L^{\infty}}\left\|\nabla \mathcal{H}\left(v^{\varepsilon}\right)\right\|_{L^{2}}\left\|\nabla v^{\varepsilon}\right\|_{L^{2}} \\
& +\left\|\nabla a^{\varepsilon}\right\|_{L^{\infty}}\left\|\mathcal{H}\left(v^{\varepsilon}\right)\right\|_{L^{2}}\left\|\nabla v^{\varepsilon}\right\|_{L^{2}} \\
& +\varepsilon\left\|v^{\varepsilon}\right\|_{L^{6}}\left\|\nabla \mathcal{H}\left(v^{\varepsilon}\right)\right\|_{L^{2}}\left\|\nabla v^{\varepsilon}\right\|_{L^{3}} \\
& \leq C\left(\left\|v^{\varepsilon}\right\|_{H^{1}}^{2}+\varepsilon\left\|v^{\varepsilon}\right\|_{H^{1}}^{\frac{5}{2}}\left\|v^{\varepsilon}\right\|_{H^{2}}^{\frac{1}{2}}\right) \\
& \leq C \mathrm{Q}+C \varepsilon^{\frac{1}{2}} \mathbf{Q}^{\frac{3}{2}} \\
& \begin{aligned}
\left|\int_{\Omega} T_{6} \Delta v^{\varepsilon}\right| \leq & 2\left\|\nabla a^{\varepsilon}\right\|_{L^{\infty}}\left\|a^{\varepsilon}\right\|_{L^{\infty}}\left\|\mathcal{H}\left(v^{\varepsilon}\right)\right\|_{L^{2}}\left\|\nabla v^{\varepsilon}\right\|_{L^{2}} \\
& +\left\|a^{\varepsilon}\right\|_{L^{\infty}}^{2}\left\|\nabla \mathcal{H}\left(v^{\varepsilon}\right)\right\|_{L^{2}}\left\|\nabla v^{\varepsilon}\right\|_{L^{2}}+\left\|\nabla a^{\varepsilon}\right\|_{L^{\infty}}\left\|v^{\varepsilon}\right\|_{L^{2}}\left\|K^{\varepsilon}\right\|_{L^{\infty}}\left\|\nabla v^{\varepsilon}\right\|_{L^{2}} \\
& +\left\|a^{\varepsilon}\right\|_{L^{\infty}}\left\|\nabla v^{\varepsilon}\right\|_{L^{2}}^{2}\left\|K^{\varepsilon}\right\|_{L^{\infty}}+2\left\|a^{\varepsilon}\right\|_{L^{\infty}}\left\|v^{\varepsilon}\right\|_{L^{3}}\left\|\nabla K^{\varepsilon}\right\|_{L^{6}}\left\|\nabla v^{\varepsilon}\right\|_{L^{2}} \\
& +\left\|v^{\varepsilon}\right\|_{L^{2}}\left\|\nabla a^{\varepsilon}\right\|_{L^{\infty}}\left\|K^{\varepsilon}\right\|_{L^{\infty}}\left\|\nabla v^{\varepsilon}\right\|_{L^{2}} \\
\leq & C\left\|v^{\varepsilon}\right\|_{H^{1}}^{2} \\
\leq & C \mathbf{Q}
\end{aligned}
\end{aligned}
$$$$
\left|\int_{\Omega} T_{7} \Delta v^{\varepsilon}\right| \leq \varepsilon\left\|v^{\varepsilon}\right\|_{L^{6}}\left\|\nabla v^{\varepsilon}\right\|_{L^{3}}\left\|\nabla v^{\varepsilon}\right\|_{L^{2}}\left\|K^{\varepsilon}\right\|_{L^{\infty}}
$$$$
+\varepsilon\left\|v^{\varepsilon}\right\|_{L^{6}}^{2}\left\|\nabla K^{\varepsilon}\right\|_{L^{6}}\left\|\nabla v^{\varepsilon}\right\|_{L^{2}}
$$$$
+2 \varepsilon\left\|\nabla a^{\varepsilon}\right\|_{L^{\infty}}\left\|v^{\varepsilon}\right\|_{L^{6}}\left\|\mathcal{H}\left(v^{\varepsilon}\right)\right\|_{L^{3}}\left\|\nabla v^{\varepsilon}\right\|_{L^{2}}
$$$$
+2 \varepsilon\left\|a^{\varepsilon}\right\|_{L^{\infty}}\left\|\nabla v^{\varepsilon}\right\|_{L^{3}}\left\|\mathcal{H}\left(v^{\varepsilon}\right)\right\|_{L^{6}}\left\|v^{\varepsilon}\right\|_{L^{2}}
$$$$
+2 \varepsilon\left\|a^{\varepsilon}\right\|_{L^{\infty}}\left\|v^{\varepsilon}\right\|_{L^{6}}\left\|\nabla \mathcal{H}\left(v^{\varepsilon}\right)\right\|_{L^{3}}\left\|\nabla v^{\varepsilon}\right\|_{L^{2}}
$$$$
\leq C\left(\varepsilon^{\frac{1}{2}}\left\|v^{\varepsilon}\right\|_{H^{1}}^{\frac{5}{2}}\left\|\varepsilon v^{\varepsilon}\right\|_{H^{2}}^{\frac{1}{2}}+\varepsilon\left\|v^{\varepsilon}\right\|_{H^{1}}^{3}+\varepsilon\left\|v^{\varepsilon}\right\|_{L^{2}}^{\frac{1}{2}}\left\|v^{\varepsilon}\right\|_{H^{1}}^{\frac{5}{2}}\right)
$$$$
\leq C \varepsilon^{\frac{1}{2}} \mathbf{Q}^{\frac{3}{2}}+C \varepsilon \mathbf{Q}^{\frac{3}{2}}
$$ 


$$
\begin{aligned}
\left|\int_{\Omega} T_{8} \Delta v^{\varepsilon}\right| \leq & \varepsilon^{2}\left\|v^{\varepsilon}\right\|_{L^{6}}\left\|\nabla v^{\varepsilon}\right\|_{L^{3}}^{2}\left\|\mathcal{H}\left(v^{\varepsilon}\right)\right\|_{L^{6}} \\
& +\varepsilon^{2}\left\|v^{\varepsilon}\right\|_{L^{6}}^{2}\left\|\nabla \mathcal{H}\left(v^{\varepsilon}\right)\right\|_{L^{3}}\left\|\nabla v^{\varepsilon}\right\|_{L^{3}} \\
\leq & C \varepsilon\left\|v^{\varepsilon}\right\|_{H^{1}}^{3}\left\|\varepsilon v^{\varepsilon}\right\|_{H^{2}} \\
\leq & C \varepsilon \mathbf{Q}^{2} \\
\left|\int_{\Omega} F^{\varepsilon} \Delta v^{\varepsilon}\right| \leq & \left\|\nabla F^{\varepsilon}\right\|_{L^{2}}\left\|\nabla v^{\varepsilon}\right\|_{L^{2}} \\
\leq & C\left(1+\left\|\nabla v^{\varepsilon}\right\|_{L^{2}}^{2}\right)
\end{aligned}
$$

Ainsi, en sommant les inégalités précédentes nous obtenons:

$$
\frac{d}{d t}\left(\left\|\nabla v^{\varepsilon}\right\|_{L^{2}}\right)+\varepsilon^{2}\left\|\Delta v^{\varepsilon}\right\|_{L^{2}}^{2} \leq C+C \mathbf{Q}+\varepsilon^{1 / 2} P(\mathbf{Q})
$$

où $P$ est un polynôme.

Estimation $H^{2}$.

Nous allons estimer $\left\|\varepsilon \Delta v^{\varepsilon}\right\|_{L^{2}}^{2}$. On multiplie l'équation par $\varepsilon^{2} \Delta^{2} v^{\varepsilon}$. Sur chaque terme obtenu, on utilise tout d'abord les inégalités de Hölder, puis les injections de Sobolev, et enfin l'inégalité de Young pour isoler le terme en $\varepsilon^{4}\left\|\nabla \Delta v^{\varepsilon}\right\|_{L^{2}}^{2}$, on obtient:

$$
\begin{aligned}
\left|\int_{\Omega} \varepsilon^{2} T_{1} \Delta^{2} v^{\varepsilon}\right| & \leq \varepsilon^{6}\left(\left\|\nabla v^{\varepsilon}\right\|_{L^{6}}^{3}\left\|\nabla \Delta v^{\varepsilon}\right\|_{L^{2}}+\left\|v^{\varepsilon}\right\|_{L^{12}}\left\|\Delta v^{\varepsilon}\right\|_{L^{3}}\left\|\nabla v^{\varepsilon}\right\|_{L^{12}}\left\|\nabla \Delta v^{\varepsilon}\right\|_{L^{2}}\right) \\
& \leq C\left(\varepsilon\left\|\varepsilon v^{\varepsilon}\right\|_{H^{2}}^{3}\left\|\varepsilon^{2} \nabla \Delta v^{\varepsilon}\right\|_{L^{2}}+\varepsilon\left\|v^{\varepsilon}\right\|_{H^{1}}^{\frac{3}{4}}\left\|\varepsilon v^{\varepsilon}\right\|_{H^{2}}^{\frac{3}{2}}\left\|\varepsilon^{2} v^{\varepsilon}\right\|_{H^{3}}^{\frac{3}{4}}\left\|\varepsilon^{2} \nabla \Delta v^{\varepsilon}\right\|_{L^{2}}\right) \\
& \leq C \varepsilon \mathbf{Q}^{\frac{3}{2}}\left\|\varepsilon^{2} \nabla \Delta v^{\varepsilon}\right\|_{L^{2}}+C \varepsilon^{\frac{7}{4}} \mathbf{Q}^{\frac{3}{2}}\left\|\varepsilon^{2} \nabla \Delta v^{\varepsilon}\right\|_{L^{2}}+C \varepsilon \mathbf{Q}^{\frac{9}{8}}\left\|\varepsilon^{2} \nabla \Delta v^{\varepsilon}\right\|_{L^{2}}^{\frac{7}{4}} \\
& \leq \frac{1}{18}\left\|\varepsilon^{2} \nabla \Delta v^{\varepsilon}\right\|_{L^{2}}^{2}+C \varepsilon^{2} \mathbf{Q}^{3}+C \varepsilon^{8} \mathbf{Q}^{9}
\end{aligned}
$$




$$
\begin{aligned}
& \left|\int_{\Omega} \varepsilon^{2} T_{2} \Delta^{2} v^{\varepsilon}\right| \leq 2 \varepsilon^{5}\left\|\nabla a^{\varepsilon}\right\|_{L^{\infty}}\left\|\nabla v^{\varepsilon}\right\|_{L^{4}}^{2}\left\|\nabla \Delta v^{\varepsilon}\right\|_{L^{2}} \\
& +\varepsilon^{5}\left\|a^{\varepsilon}\right\|_{L^{\infty}}\left\|D^{2} v^{\varepsilon}\right\|_{L^{3}}\left\|\nabla v^{\varepsilon}\right\|_{L^{6}}\left\|\nabla \Delta v^{\varepsilon}\right\|_{L^{2}} \\
& +\varepsilon^{5}\left\|v^{\varepsilon}\right\|_{L^{12}}\left\|D^{2} a^{\varepsilon}\right\|_{L^{12}}\left\|\nabla v^{\varepsilon}\right\|_{L^{3}}\left\|\nabla \Delta v^{\varepsilon}\right\|_{L^{2}} \\
& +\varepsilon^{5}\left\|v^{\varepsilon}\right\|_{L^{6}}\left\|\nabla a^{\varepsilon}\right\|_{L^{\infty}}\left\|D^{2} v^{\varepsilon}\right\|_{L^{3}}\left\|\nabla \Delta v^{\varepsilon}\right\|_{L^{2}} \\
& \leq C \varepsilon^{5}\left\|v^{\varepsilon}\right\|_{H_{5}^{1}}^{\frac{1}{2}}\left\|v^{\varepsilon}\right\|_{H^{2}}^{\frac{3}{2}}\left\|\nabla \Delta v^{\varepsilon}\right\|_{L^{2}}+C \varepsilon^{5}\left\|v^{\varepsilon}\right\|_{H^{2}}^{\frac{3}{2}}\left\|v^{\varepsilon}\right\|_{H_{1}^{3}}^{\frac{1}{2}}\left\|\nabla \Delta v^{\varepsilon}\right\|_{L^{2}} \\
& +C \varepsilon^{4}\left\|v^{\varepsilon}\right\|_{H^{1}}^{\frac{5}{4}}\left\|v^{\varepsilon}\right\|_{H^{2}}^{\frac{3}{4}}\left\|\nabla \Delta v^{\varepsilon}\right\|_{L^{2}}+C \varepsilon^{5}\left\|v^{\varepsilon}\right\|_{H^{1}}\left\|v^{\varepsilon}\right\|_{H^{2}}^{\frac{1}{2}}\left\|v^{\varepsilon}\right\|_{H^{3}}^{\frac{1}{2}}\left\|\nabla \Delta v^{\varepsilon}\right\|_{L^{2}} \\
& \leq C \varepsilon^{\frac{5}{4}} \mathbf{Q}\left\|\varepsilon^{2} \nabla \Delta v^{\varepsilon}\right\|_{L^{2}}+C \varepsilon^{\frac{3}{2}} \mathbf{Q}^{\frac{3}{2}}\left\|\varepsilon^{2} \nabla \Delta v^{\varepsilon}\right\|_{L^{2}}^{\frac{3}{2}} \\
& \leq \frac{1}{18}\left\|\varepsilon^{2} \nabla \Delta v^{\varepsilon}\right\|_{L^{2}}^{2}+C \varepsilon^{\frac{5}{2}} \mathbf{Q}^{2}+C \varepsilon^{6} \mathbf{Q}^{6} \\
& \left|\int_{\Omega} \varepsilon^{2} T_{3} \Delta^{2} v^{\varepsilon}\right| \leq 2 \varepsilon^{4}\left\|\nabla v^{\varepsilon}\right\|_{L^{2}}\left\|\nabla a^{\varepsilon}\right\|_{L^{\infty}}^{2}\left\|\nabla \Delta v^{\varepsilon}\right\|_{L^{2}} \\
& +\varepsilon^{4}\left\|v^{\varepsilon}\right\|_{L^{2}}\left\|\nabla a^{\varepsilon}\right\|_{L^{\infty}}\left\|D^{2} a^{\varepsilon}\right\|_{L^{\infty}}\left\|\nabla \Delta v^{\varepsilon}\right\|_{L^{2}} \\
& +\varepsilon^{4}\left\|a^{\varepsilon}\right\|_{L^{\infty}}\left\|\nabla a^{\varepsilon}\right\|_{L^{\infty}}\left\|D^{2} v^{\varepsilon}\right\|_{L^{2}}\left\|\nabla \Delta v^{\varepsilon}\right\|_{L^{2}} \\
& +\varepsilon^{4}\left\|a^{\varepsilon}\right\|_{L^{\infty}}\left\|\nabla v^{\varepsilon}\right\|_{L^{2}}\left\|D^{2} a^{\varepsilon}\right\|_{L^{\infty}}\left\|\nabla \Delta v^{\varepsilon}\right\|_{L^{2}} \\
& \leq \varepsilon^{4}\|\nabla v\|_{L^{2}}\left\|\nabla \Delta v^{\varepsilon}\right\|_{L^{2}}+\varepsilon^{3}\left\|v^{\varepsilon}\right\|_{L^{2}}\left\|\nabla \Delta v^{\varepsilon}\right\|_{L^{2}}+C \varepsilon^{4}\left\|v^{\varepsilon}\right\|_{H^{2}}\left\|\nabla \Delta v^{\varepsilon}\right\|_{L^{2}} \\
& +C \varepsilon^{3}\left\|v^{\varepsilon}\right\|_{H^{1}}\left\|\nabla \Delta v^{\varepsilon}\right\|_{L^{2}} \\
& \leq C \varepsilon \mathbf{Q}\left\|\nabla \Delta v^{\varepsilon}\right\|_{L^{2}} \\
& \leq \frac{1}{18}\left\|\varepsilon^{2} \nabla \Delta v^{\varepsilon}\right\|_{L^{2}}^{2}+C \varepsilon^{2} \mathbf{Q}^{2} \\
& \left|\int_{\Omega} \varepsilon^{2} T_{4} \Delta^{2} v^{\varepsilon}\right| \leq \varepsilon^{3}\left\|\nabla v^{\varepsilon}\right\|_{L^{2}}\left\|\Delta a^{\varepsilon}\right\|_{L^{\infty}}\left\|\nabla \Delta v^{\varepsilon}\right\|_{L^{2}} \\
& +\varepsilon^{4}\left\|v^{\varepsilon}\right\|_{L^{6}}\left\|\nabla \Delta a^{\varepsilon}\right\|_{L^{3}}\left\|\nabla \Delta v^{\varepsilon}\right\|_{L^{2}} \\
& +\varepsilon^{4}\left\|\Delta v^{\varepsilon}\right\|_{L^{2}}\left\|\nabla a^{\varepsilon}\right\|_{L^{\infty}}\left\|\nabla \Delta v^{\varepsilon}\right\|_{L^{2}} \\
& +\varepsilon^{5}\left\|\nabla v^{\varepsilon}\right\|_{L^{6}}\left\|\Delta v^{\varepsilon}\right\|_{L^{3}}\left\|\nabla \Delta v^{\varepsilon}\right\|_{L^{2}} \\
& \leq C \varepsilon^{3}\left\|v^{\varepsilon}\right\|_{H^{1}}\left\|\nabla \Delta v^{\varepsilon}\right\|_{L^{2}}+C \varepsilon^{2}\left\|v^{\varepsilon}\right\|_{H^{1}}\left\|\nabla \Delta v^{\varepsilon}\right\|_{L^{2}} \\
& +C \varepsilon^{4}\left\|v^{\varepsilon}\right\|_{H^{2}}\left\|\nabla \Delta v^{\varepsilon}\right\|_{L^{2}}+C \varepsilon^{5}\left\|v^{\varepsilon}\right\|_{H^{2}}^{\frac{3}{2}}\left\|v^{\varepsilon}\right\|_{H^{3}}^{\frac{1}{2}}\left\|\nabla \Delta v^{\varepsilon}\right\|_{L^{2}} \\
& \leq C \mathbf{Q}^{\frac{1}{2}}\left\|\varepsilon^{2} \nabla \Delta v^{\varepsilon}\right\|_{L^{2}}+C \varepsilon \mathbf{Q}\left\|\varepsilon^{2} \nabla \Delta v^{\varepsilon}\right\|_{L^{2}}+C \varepsilon^{\frac{1}{2}} \mathbf{Q}^{\frac{3}{2}}\left\|\varepsilon^{2} \nabla \Delta v^{\varepsilon}\right\|_{L^{2}}^{\frac{3}{2}} \\
& \left|\int_{\Omega} \varepsilon^{2} T_{4} \Delta^{2} v^{\varepsilon}\right| \leq \frac{1}{18}\left\|\varepsilon^{2} \nabla \Delta v^{\varepsilon}\right\|_{L^{2}}^{2}+C \mathbf{Q}+C \varepsilon^{2} \mathbf{Q}^{2}+C \varepsilon^{2} \mathbf{Q}^{6}
\end{aligned}
$$




$$
\begin{aligned}
& \left|\int_{\Omega} \varepsilon^{2} T_{5} \Delta^{2} v^{\varepsilon}\right| \leq \varepsilon^{2}\left\|\nabla v^{\varepsilon}\right\|_{L^{2}}\left\|K^{\varepsilon}\right\|_{L^{\infty}}\left\|\nabla \Delta v^{\varepsilon}\right\|_{L^{2}} \\
& +\varepsilon^{2}\left\|v^{\varepsilon}\right\|_{L^{6}}\left\|\nabla K^{\varepsilon}\right\|_{L^{3}}\left\|\nabla \Delta v^{\varepsilon}\right\|_{L^{2}} \\
& +\varepsilon^{2}\left\|\nabla a^{\varepsilon}\right\|_{L^{\infty}}\left\|\mathcal{H}\left(v^{\varepsilon}\right)\right\|_{L^{2}}\left\|\nabla \Delta v^{\varepsilon}\right\|_{L^{2}} \\
& +\varepsilon^{2}\left\|a^{\varepsilon}\right\|_{L^{\infty}}\left\|\nabla \mathcal{H}\left(v^{\varepsilon}\right)\right\|_{L^{2}}\left\|\nabla \Delta v^{\varepsilon}\right\|_{L^{2}} \\
& +\varepsilon^{3}\left\|\nabla v^{\varepsilon}\right\|_{L^{3}}\left\|\mathcal{H}\left(v^{\varepsilon}\right)\right\|_{L^{6}}\left\|\nabla \Delta v^{\varepsilon}\right\|_{L^{2}} \\
& +\varepsilon^{3}\left\|v^{\varepsilon}\right\|_{L^{6}}\left\|\nabla \mathcal{H}\left(v^{\varepsilon}\right)\right\|_{L^{3}}\left\|\nabla \Delta v^{\varepsilon}\right\|_{L^{2}} \\
& \leq C \varepsilon^{2}\left\|v^{\varepsilon}\right\|_{H^{1}}\left\|\nabla \Delta v^{\varepsilon}\right\|_{L^{2}}+C \varepsilon^{3}\left\|v^{\varepsilon}\right\|_{H^{1}}^{\frac{3}{2}}\left\|v^{\varepsilon}\right\|_{H^{2}}^{\frac{1}{2}}\left\|\nabla \Delta v^{\varepsilon}\right\|_{L^{2}} \\
& \leq C \mathbf{Q}^{\frac{1}{2}}\left\|\varepsilon^{2} \nabla \Delta v^{\varepsilon}\right\|_{L^{2}}+C \varepsilon^{\frac{1}{2}} \mathbf{Q}\left\|\varepsilon^{2} \nabla \Delta v^{\varepsilon}\right\|_{L^{2}} \\
& \leq \frac{1}{18}\left\|\varepsilon^{2} \nabla \Delta v^{\varepsilon}\right\|_{L^{2}}^{2}+C \mathbf{Q}+C \varepsilon \mathbf{Q}^{2} \\
& \left|\int_{\Omega} \varepsilon^{2} T_{6} \Delta^{2} v^{\varepsilon}\right| \leq 2 \varepsilon^{2}\left\|\nabla a^{\varepsilon}\right\|_{L^{\infty}}\left\|a^{\varepsilon}\right\|_{L^{\infty}}\left\|\mathcal{H}\left(v^{\varepsilon}\right)\right\|_{L^{2}}\left\|\nabla \Delta v^{\varepsilon}\right\|_{L^{2}} \\
& +\varepsilon^{2}\left\|a^{\varepsilon}\right\|_{L^{\infty}}^{2}\left\|\nabla \mathcal{H}\left(v^{\varepsilon}\right)\right\|_{L^{2}}\left\|\nabla \Delta v^{\varepsilon}\right\|_{L^{2}} \\
& +\varepsilon^{2}\left\|\nabla a^{\varepsilon}\right\|_{L^{\infty}}\left\|v^{\varepsilon}\right\|_{L^{2}}\left\|K^{\varepsilon}\right\|_{L^{\infty}}\left\|\nabla \Delta v^{\varepsilon}\right\|_{L^{2}} \\
& +\varepsilon^{2}\left\|a^{\varepsilon}\right\|_{L^{\infty}}\left\|\nabla v^{\varepsilon}\right\|_{L^{2}}\left\|K^{\varepsilon}\right\|_{L^{\infty}}\left\|\nabla \Delta v^{\varepsilon}\right\|_{L^{2}} \\
& +\varepsilon^{2}\left\|a^{\varepsilon}\right\|_{L^{\infty}}\left\|v^{\varepsilon}\right\|_{L^{6}}\left\|\nabla K^{\varepsilon}\right\|_{L^{3}}\left\|\nabla \Delta v^{\varepsilon}\right\|_{L^{2}} \\
& \leq \varepsilon^{2}\left\|v^{\varepsilon}\right\|_{L^{2}}\left\|\nabla \Delta v^{\varepsilon}\right\|_{L^{2}}+C \varepsilon^{2}\left\|v^{\varepsilon}\right\|_{H^{1}}\left\|\nabla \Delta v^{\varepsilon}\right\|_{L^{2}} \\
& \leq C \mathbf{Q}^{\frac{1}{2}}\left\|\varepsilon^{2} \nabla \Delta v^{\varepsilon}\right\|_{L^{2}} \\
& \leq \frac{1}{18}\left\|\varepsilon^{2} \nabla \Delta v^{\varepsilon}\right\|_{L^{2}}^{2}+C \mathbf{Q} \\
& \left|\int_{\Omega} \varepsilon^{2} T_{7} \Delta^{2} v^{\varepsilon}\right| \leq \varepsilon^{3}\left\|v^{\varepsilon}\right\|_{L^{6}}\left\|\nabla v^{\varepsilon}\right\|_{L^{3}}\left\|K^{\varepsilon}\right\|_{L^{\infty}}\left\|\nabla \Delta v^{\varepsilon}\right\|_{L^{2}} \\
& +\varepsilon^{3}\left\|v^{\varepsilon}\right\|_{L^{6}}\left\|v^{\varepsilon}\right\|_{L^{6}}\left\|\nabla K^{\varepsilon}\right\|_{L^{6}}\left\|\nabla \Delta v^{\varepsilon}\right\|_{L^{2}} \\
& +\varepsilon^{3}\left\|v^{\varepsilon}\right\|_{L^{6}}\left\|\nabla a^{\varepsilon}\right\|_{L^{\infty}}\left\|\mathcal{H}\left(v^{\varepsilon}\right)\right\|_{L^{3}}\left\|\nabla \Delta v^{\varepsilon}\right\|_{L^{2}} \\
& +\varepsilon^{3}\left\|a^{\varepsilon}\right\|_{L^{\infty}}\left\|\nabla v^{\varepsilon}\right\|_{L^{3}}\left\|\mathcal{H}\left(v^{\varepsilon}\right)\right\|_{L^{6}}\left\|\nabla \Delta v^{\varepsilon}\right\|_{L^{2}} \\
& +\varepsilon^{3}\left\|a^{\varepsilon}\right\|_{L^{\infty}}\left\|v^{\varepsilon}\right\|_{L^{6}}\left\|\nabla \mathcal{H}\left(v^{\varepsilon}\right)\right\|_{L^{3}}\left\|\nabla \Delta v^{\varepsilon}\right\|_{L^{2}} \\
& \leq C \varepsilon^{3}\left\|v^{\varepsilon}\right\|_{H^{1}}^{\frac{3}{2}}\left\|v^{\varepsilon}\right\|_{H^{2}}^{\frac{1}{2}}\left\|\nabla \Delta v^{\varepsilon}\right\|_{L^{2}}+C \varepsilon^{3}\left\|v^{\varepsilon}\right\|_{H^{1}}^{2}\left\|\nabla \Delta v^{\varepsilon}\right\|_{L^{2}} \\
& +C \varepsilon^{3}\left\|v^{\varepsilon}\right\|_{H^{1}}^{\frac{3}{2}}\left\|v^{\varepsilon}\right\|_{L^{2}}^{\frac{1}{2}}\left\|\nabla \Delta v^{\varepsilon}\right\|_{L^{2}} \\
& \leq C \varepsilon^{\frac{1}{2}} \mathbf{Q}\left\|\varepsilon^{2} \nabla \Delta v^{\varepsilon}\right\|_{L^{2}} \\
& \leq \frac{1}{18}\left\|\varepsilon^{2} \nabla \Delta v^{\varepsilon}\right\|_{L^{2}}^{2}+C \varepsilon \mathbf{Q}^{2}
\end{aligned}
$$




$$
\begin{aligned}
\left|\int_{\Omega} \varepsilon^{2} T_{8} \Delta^{2} v^{\varepsilon}\right| \leq & 2 \varepsilon^{4}\left\|v^{\varepsilon}\right\|_{L^{6}}\left\|\nabla v^{\varepsilon}\right\|_{L^{6}}\left\|\mathcal{H}\left(v^{\varepsilon}\right)\right\|_{L^{6}}\left\|\nabla \Delta v^{\varepsilon}\right\|_{L^{2}} \\
& +\varepsilon^{4}\left\|v^{\varepsilon}\right\|_{L^{6}}^{2}\left\|\nabla \mathcal{H}\left(v^{\varepsilon}\right)\right\|_{L^{2}}\left\|\nabla \Delta v^{\varepsilon}\right\|_{L^{2}} \\
\leq & C \varepsilon^{4}\left\|v^{\varepsilon}\right\|_{H^{1}}^{2}\left\|v^{\varepsilon}\right\|_{H^{2}}\left\|\nabla \Delta v^{\varepsilon}\right\|_{L^{2}} \\
\leq & C \varepsilon \mathbf{Q}^{\frac{3}{2}}\left\|\varepsilon^{2} \nabla \Delta v^{\varepsilon}\right\|_{L^{2}} \\
\leq & \frac{1}{18}\left\|\varepsilon^{2} \nabla \Delta v^{\varepsilon}\right\|_{L^{2}}^{2}+C \varepsilon^{2} \mathrm{Q}^{3} \\
\left|\int_{\Omega} \varepsilon^{2} F^{\varepsilon} \Delta^{2} v^{\varepsilon}\right| \leq & \varepsilon^{2}\left\|\nabla F^{\varepsilon}\right\|_{L^{2}}\left\|\nabla \Delta v^{\varepsilon}\right\|_{L^{2}} \\
\leq & C\left\|\varepsilon^{2} \nabla \Delta v^{\varepsilon}\right\|_{L^{2}} \\
\leq & \frac{1}{18}\left\|\varepsilon^{2} \nabla \Delta v^{\varepsilon}\right\|_{L^{2}}^{2}+C
\end{aligned}
$$

En sommant les inégalités précédentes, on obtient :

$$
\frac{d}{d t}\left(\left\|\varepsilon \Delta v^{\varepsilon}\right\|_{L^{2}}^{2}\right)+\varepsilon^{2}\left\|\varepsilon \nabla \Delta v^{\varepsilon}\right\|_{L^{2}}^{2} \leq C(1+\mathbf{Q})+\varepsilon P(\mathbf{Q})
$$

où $P$ est un polynôme de degré 6 indépendant de $\varepsilon$.

En sommant les inégalités (6.39), (6.38), (6.37) nous obtenons finalement :

$$
\frac{d \mathbf{Q}}{d t}+\varepsilon^{2}\left(\left\|\nabla v^{\varepsilon}\right\|_{L^{2}}^{2}+\left\|\Delta v^{\varepsilon}\right\|_{L^{2}}^{2}+\left\|\varepsilon \nabla \Delta v^{\varepsilon}\right\|_{L^{2}}^{2}\right) \leq C(1+\mathbf{Q})+\varepsilon^{1 / 2} P(\mathbf{Q})
$$

ce qui entraine l'inégalité (6.36). De façon classique, on en déduit qu'il existe un temps $T_{\varepsilon}$, vérifiant $\lim _{\varepsilon \rightarrow 0} T_{\varepsilon}=+\infty$ tel que $v^{\varepsilon}$ soit borné indépendament de $\varepsilon$ dans $L^{\infty}\left(0, T ; H^{1}(\Omega)\right)$, et $\varepsilon v^{\varepsilon}$ soit borné indépendament de $\varepsilon$ dans $L^{\infty}\left(0, T ; H^{2}(\Omega)\right)$ pour tout $T$.

\section{REMERCIEMENTS}

Les auteurs remercient chaleureusement Jean-Luc Joly et Guy Métivier pour l'intérêt qu'ils ont porté à ce travail 


\section{REFERENCES}

[1] R. A. Adams, Sobolev space, Pure and Applied Math. 65, Academic press 1975.

[2] S. Agmon, Elliptic boundary values problems, Van Nostrand Company, 1965

[3] C. Bardos, J. Rauch, Maximal positive boundary value problems as limits of singular perturbation problems, Trans. Amer. Math. Soc., 270 , (1982), $377-408$.

[4] F. Brown, Micromagnetics, Wiley, New York, 1963.

[5] L. Boutet de Monvel, Comportement d'un opérateur pseudodifférentiel sur une variété à bord, J. Anal. Math. 17 (1966), 241-304.

[6] G. Carbou, Thin layers in Micromagnetism, à paraître dans M3AS.

[7] G. Carbou, P. Fabrie, Time average in Micromagnetism, Journal of Differential Equations $14 \mathbf{7}$ (1998), 383-409.

[8] G. Carbou, P. Fabrie, Regular Solutions for Landau-Lifschitz Equation in a Bounded Domain, Differential and Integral Equations, 14 (2001), 213-229.

[9] J. Chazarain, A. Piriou, Introduction à la théorie des équations aux dérivées partielles linéaires, Gauthiers-Villars, 1981.

[10] G. Foias, R. Temam, Remarques sur les équations de Navier-Stokes stationnaires et les phénomènes successifs de bifurcation, Ann. Scuola Norm. Super. Pisa IV, $\underline{\mathbf{5}}$ (1978), 29-63.

[11] M. Gisclon,Etude des conditions aux limites pour un système strictement hyperbolique, via l'approximation parabolique, J. Math. Pures Appl. $\underline{\mathbf{7 5}}$, (1996), 485-508.

[12] E. Grenier, O. Guès, Boundary layers for viscous perturbations of noncharacteristic quasilinear hyperbolic problems, J. Differential Equations, $\underline{143}$ (1998), 110-146.

[13] O. Guès, Perturbations visqueuses de problèmes mixtes hyperboliques et couches limites, Ann. Inst. Fourier, Grenoble, $\underline{45}$ (1995), 973-1006.

[14] H. Haddar, Thèse de l'Ecole Nationale des Ponts et Chaussées (2000). 
[15] H. Haddar, P. Joly, Effective boundary conditions for thin ferromagnetic layers ; the 1d model, Siam J. Appl. Math., à paraître.

[16] L. Hörmander, The analysis of linear Partial Differential Operators III, Springer-Verlag, 1985.

[17] J.-L. Joly, G. Métivier, J. Rauch, Global solutions to Maxwell equations in a ferromagnetic medium, Ann. Henri Poincaré 1 (2000), $\underline{\mathbf{2}}$, 307-340

[18] P. Joly, O. Vacus, Mathematical and numerical studies of nonlinear ferromagnetic materials. M2AN Math. Model. Numer. Anal. $\underline{33}$ (1999), 593-626.

[19] S. Labbé et P.Y. Bertin, Microwave polarizability of ferrite particles, Journal of Magnetism and Magnetic Materials, _206 (1999), 93-105.

[20] S. Labbé, Simulation numérique du comportement hyperfréquence des matériaux ferromagnétiques, Thèse de l'Université Paris 13 (1998).

[21] O. A. Ladysenskaya, The boundary value problem of mathematical physics, Springer Verlag Applied Math. Sciences, 또, 1985

[22] L. Landau et E. Lifschitz, Electrodynamique des milieux continues, cours de physique théorique, tome VIII (ed. Mir) Moscou, 1969.

[23] J.-L. Lions, Perturbations singulières dans les problèmes aux limites et en contrôle optimal, "Lecture Notes in Math.", 323, Springer-Verlag, 1973.

[24] G. Métivier, communication privée.

[25] D. Sanchez, Un exemple de couches limites en ferromagnétisme, en préparation

[26] D. Serre, Systèmes de lois de conservation II, Diderot éditeur, Art et Sciences, 1996.

[27] A. Visintin, On Landau Lifschitz equation for ferromagnetism, Japan Journal of Applied Mathematics, 1 (1985), 69-84.

[28] H. Wynled, Ferromagnetism, Encyclopedia of Physics, Vol. XVIII / 2. Springer Verlag, Berlin, 1966. 